\title{
Entanglement measures of bipartite quantum gates and their thermalization under arbitrary interaction strength
}

\author{
Bhargavi Jonnadula, ${ }^{1}$ Prabha Mandayam, ${ }^{2}$ Karol Życzkowski, ${ }^{3,4}$ and Arul Lakshminarayan ${ }^{2,5}$ \\ ${ }^{1}$ School of Mathematics, University of Bristol, BS8 1UG, United Kingdom \\ ${ }^{2}$ Department of Physics, Indian Institute of Technology Madras, 600036 Chennai, India \\ ${ }^{3}$ Institute of Theoretical Physics, Jagiellonian University, 30-348 Cracow, Poland \\ ${ }^{4}$ Center for Theoretical Physics, Polish Academy of Sciences, 02-668 Warsaw, Poland \\ ${ }^{5}$ Max-Planck-Institut für Physik komplexer Systeme, Nöthnitzer Strasse 38, 01187 Dresden, Germany
}

(Received 5 October 2019; accepted 30 September 2020; published 23 October 2020)

\begin{abstract}
Entanglement properties of bipartite unitary operators are studied via their local invariants, namely the entangling power $e_{p}$ and a complementary quantity, the gate typicality $g_{t}$. We characterize the boundaries of the set $K_{2}$ representing all two-qubit gates projected onto the plane $\left(e_{p}, g_{t}\right)$ showing that the fractional powers of the SWAP operator form a parabolic boundary of $K_{2}$, while the other bounds are formed by two straight lines. In this way, a family of gates with extreme properties is identified and analyzed. We also show that the parabolic curve representing powers of SWAP persists in the set $K_{N}$ for gates of higher dimensions $(N>2)$. Furthermore, we study entanglement of bipartite quantum gates applied sequentially $n$ times, and we analyze the influence of interlacing local unitary operations, which model generic Hamiltonian dynamics. An explicit formula for the entangling power of a gate applied $n$ times averaged over random local unitary dynamics is derived for an arbitrary dimension of each subsystem. This quantity shows an exponential saturation to the value predicted by the random matrix theory, indicating "thermalization" in the entanglement properties of sequentially applied quantum gates that can have arbitrarily small, but nonzero, entanglement to begin with. The thermalization is further characterized by the spectral properties of the reshuffled and partially transposed unitary matrices.
\end{abstract}

DOI: 10.1103/PhysRevResearch.2.043126

\section{INTRODUCTION}

A clutch of quantities such as state entanglement, operator entanglement, operator scrambling, out-of-time-ordered correlators, and various measures of mutual information are currently being actively pursued as a means to understand information transport in complex quantum systems and to characterize quantum chaos [1-8]. The entangling power of the time-evolution operator has been studied since its introduction as a state-independent measure [9-11], and it is the average entanglement an operator produces when acting on product, unentangled, states. On the other hand, operator entanglement quantifies to what extent a given operator, treated as a vector in the Hilbert-Schmidt space of operators, is close to the tensor product [12]. Operator entanglement and entangling power have both been applied recently to many-body systems, in particular in the context of spin-chains and conformal field theories $[4,13,14]$, where it has been found useful to distinguish between integrable and nonintegrable systems as well as in the analysis of the many-body-localization transition.

Published by the American Physical Society under the terms of the Creative Commons Attribution 4.0 International license. Further distribution of this work must maintain attribution to the author(s) and the published article's title, journal citation, and DOI.
The study of the operator entanglement and entangling power of the time-evolution operator $\exp (-i H t / \hbar)$, or its time-ordered version if the Hamiltonian $H$ is a function of time, is of fundamental import in the growth of subsystem entropy and complexity of closed systems ranging from two large bipartite systems to quantum spins on lattices [15-32]. The action of this time-evolution operator on unentangled states generally creates multipartite entanglement. From another perspective, the Heisenberg evolution results in operator entanglement and scrambling in the space of operators $[2,7,8,33]$. A central aspect of this paper is the study of the dynamics of quantum entanglement in products of unitary matrices, which are interpreted as time-evolution operators, with the number of terms in the product playing the role of discrete time.

From the point of view of quantum computing [34], gate operations ordered in time are the source of information transfer. Products of unitary operators are therefore natural objects to study as they form building blocks for quantum algorithms. Random quantum circuits with random unitary operators providing interaction among qubits have been studied in this context [35-37]. They are known to be approximate unitary $t$-designs that simulate Haar distributed unitaries $[38,39]$. Models of random quantum circuits have been studied in many other contexts including randomized benchmarking [40], entanglement spreading, scrambling, and many-body localization $[7,8,41]$. 
Random quantum circuits are constructed by arbitrarily choosing pairs of quNits between which the interactions are described by random unitary matrices [42], typically Haar distributed. In a departure from this standard formalism, we are primarily interested in the role of local random unitaries with a tensor product structure, which interlace sequential dynamics described by a fixed nonlocal gate $V$ acting on a bipartite system. Such a bipartite structure could form a building block for more general random quantum circuits with fixed, possibly atypical, nonlocal gates and random or generic local interaction.

Another setting in which products of nonlocal unitaries interspersed with local ones arise naturally is in kicked systems, which are being used extensively. In this context, the object of interest could be powers of the Floquet operator $U[4,14,17,19,20]$, or if the local Hamiltonians are nonautonomous, products of propagators across consecutive periods of the kicking. In particular, let

$$
H=H_{A}(t) \otimes \mathbb{1}_{B}+\mathbb{1}_{A} \otimes H_{B}(t)+H_{A B} \sum_{n=-\infty}^{\infty} \delta(t / \tau-n)
$$

be the Hamiltonian, and

$$
u_{A j}=\mathcal{T} e^{-i \int_{(j-1) \tau}^{j \tau} H_{A}(t) d t},
$$

where $\mathcal{T}$ denotes time ordering. The time-evolution operator between (just before) kicks $j-1$ and $j$ is

$$
\mathcal{U}_{j}=\left(u_{A j} \otimes u_{B j}\right) U,
$$

where $U=e^{-i \tau H_{A B}}$ arises from the nonlocal interaction at time $j \tau$, and $u_{B j}$ is defined similar to $u_{A j}$. This is the Floquet operator across the time period $\tau$ between the $j-1$ and $j$ th kicks. The propagator across $n$ kicks is

$$
\mathcal{U}^{(n)}=\mathcal{T} \prod_{j=1}^{n} \mathcal{U}_{j}=\mathcal{T} \prod_{j=1}^{n}\left(u_{A_{j}} \otimes u_{B_{j}}\right) U .
$$

The brackets around the "power" $n$ indicate that there are $n$ different terms in the product generally, and the time ordering will be assumed below and hence not explicitly indicated. Most systems that have been studied are such the $u_{A j}$ and $u_{B j}$ are independent of $j$, that is, the local Hamiltonians are autonomous. This leads to a time-periodic system with $\mathcal{U}$ as the Floquet operator, and we are then interested in the powers $\mathcal{U}^{n}$. However, a product of unitary operators with different local operators occurs in contexts such as time-dependent quenches; see, e.g., the study [43] for quenched kicked Ising spin chains.

Past work has shown that while bipartite local unitaries $u_{A} \otimes u_{B}$ have no entangling power, layering or interspersing them in time with entangling gates provides a crucial role for random local unitaries $[44,45]$. Local unitary gates are easier to apply in an experiment and are thus naturally "cheaper" than nonlocal entangling gates. However, the role of such local unitary gates in creating Haar random unitaries or in achieving thermalization to our knowledge has not been sufficiently explored. Specifically, we focus here on the thermalization of the entangling power of the unitary operator $\mathcal{U}^{(n)}$, as defined in Eq. (4), where thermalization in understood to mean that after a certain number $n$ of interaction times, the quantities studied reach the typical values corresponding to the Haar average over the unitary group. We find that the natural quantities to study are indeed the entangling power, $e_{p}$, and a complementary quantity defined in [44], as the "gate typicality," $g_{t}$. In particular, we are interested in the entangling power and gate typicality of $\mathcal{U}^{(n)}$. The importance of the operator entanglement and entangling power stems from their invariance under local unitary operations and hence measures the essential nonlocal content of the process.

This paper contains two complementary but in some ways distinct motivations and results, which for the sake of the reader's convenience we enumerate below:

(i) The first part of this paper is dedicated to visualizing the entanglement landscape of bipartite gates in dimensions $N^{2}$ in terms of entangling power and gate typicality. For the case of qubits, $N=2$, the picture is complete and we show in detail the various gates that make up the "phase-space" spanned by these two local invariants. We prove the existence of a boundary consisting exclusively of the fractional powers of the SWAP operator. Of special interest are gates that maximize entangling power, in the sense that they attain bounds set by the dimensionality $N$. It is known that for two-qubit systems, such a gate does not exist [46], while the maximal entangling power is attained for CNOT and related gates $[9,44]$.

(ii) If the first part is about the "kinematics" of the entangling power and gate typicality, the second is a study of its "dynamics" via the entangling power of the products of unitaries. We generalize earlier results [44] for equal subsystem dimensionality to the important case when the two subsystems could be of different dimensions. In a central result in this context, we demonstrate the exponentially fast thermalization of the average entangling power of $\mathcal{U}^{(n)}=\Pi_{j=1}^{n} \mathcal{U}_{j}$ with time to that of a typical unitary operator. Furthermore, we show that there are signatures of such a thermalization in the spectra of the operators obtained by reshuffling and partial transposition (both permutations) of the time-evolution operator.

Thus the second part of this work shows the thermalization of the entangling power of $U$ under time evolution with nonautonomous local evolutions. Such exponential saturation also seems to provide excellent approximations in the case of autonomous Floquet systems [44] whose dimensions are not very small, although the circumstances under which this holds need further investigation. Thus we expect applications not only for coupled chaotic systems such as the kicked top and the kicked rotor, but also to many-body systems such as the kicked and tilted field Ising models [4,14]. The exponential approach of the average entangling power of $\mathcal{U}^{(n)}$ to the Haar average is determined solely by $e_{p}(U)$, the entangling power of the interaction. This demonstrates that any nonzero value of the entangling power, however small, is sufficient to thermalize its powers interspersed with random local operators.

Apart from the entangling power, the gate typicality [44] also has a simple exponential approach to the global RMT average, depending solely upon $g_{t}(U)$, the gate typicality of the interaction. In fact, this formed an important basis for the introduction of this quantity that is naturally singled out. In contrast, the thermalization of other local invariants, such as the operator entanglement, is in the form of sums of exponentials with different rates. The extremal values of the gate-typicality, $g_{t}=0$ and 1 , correspond to local gates 
and the SWAP operator, respectively, while the average value, $g_{t}=1 / 2$ (for equal subsystem dimensions), characterizes the Haar average over the entire set of bipartite unitary gates. Thus the entangling power and gate typicality are local invariants associated with the interaction $U$ that determine the complexity of products such as $\Pi_{j=1}^{n} \mathcal{U}_{j}$.

The paper is organized as follows. Section II will introduce in detail all the relevant quantities, including operator entanglement and entangling power. Section III studies the allowed region of the invariants for the case of two qubits. We study this via the entangling power, gate typicality $\left(e_{p}, g_{t}\right)$ phase space, and we establish the boundaries of the allowed gates. Section IV discusses some special gates such as the Fourier and the fractional powers of SWAP in arbitrary dimensions, and it provides partial results for qutrits as well as conjecture that the fractional powers of SWAP form a boundary for all quNits. Finally, in Sec. V we study time evolution and prove the thermalization of entangling power (and gate typicality) under certain conditions. Here, we generalize our earlier result obtained in [44], referring to Appendix C for an elegant proof. Finally, we provide examples wherein the thermalization can be seen via the approach of the spectra of the partially transposed and reshuffled operators to the Girko circular law [47] and their squared singular values to the Marcenko-Pastur distribution [48]. Section VI provides a summary and outlook.

\section{LOCAL INVARIANTS OF OPERATORS: ENTANGLING POWER AND GATE TYPICALITY}

\section{A. Two sets of local unitary invariants and operator entanglement}

Consider a unitary operator $U$ acting on the bipartite space $\mathcal{H}_{N}^{A} \otimes \mathcal{H}_{N}^{B}$ of two parts labeled $A$ and $B$. For simplicity, we restrict our attention to spaces whose dimensions are equal (and to $N$ ). The generalization to unequal dimensions is treated in Appendix A. Operators such as $U$ may be "gates" in the language of quantum circuits, or just quantum propagators describing evolution over some finite time. The fact that $U$ need not be of a product form $u_{A} \otimes u_{B}$, with $u_{A, B}$ acting on $\mathcal{H}_{N}^{A, B}$ in general, implies that it is usually capable of creating entanglement when it acts on unentangled states. Let the operator Schmidt decomposition of $U$ be [12]

$$
U=\sum_{j=1}^{N^{2}} \sqrt{\lambda_{j}} M_{A_{j}} \otimes M_{B_{j}}
$$

where the operators on the individual spaces $M_{A_{j}}$ and $M_{B_{j}}$ are in general not unitary themselves, but they form an orthonormal basis for operators on their respective spaces, $\operatorname{tr}\left(M_{A_{j}}^{\dagger} M_{A_{k}}\right)=\operatorname{tr}\left(M_{B_{j}}^{\dagger} M_{B_{k}}\right)=\delta_{j k}$, where $\delta_{j k}$ is the Kronecker delta. The Schmidt vector $\lambda=\left\{\lambda_{j}\right\}_{j=1}^{N^{2}}$ is determined by singular values of the reshuffled matrix $U^{R}$-see Appendix A. Note that $\lambda$ is invariant under local unitary operations. Unitarity of $U$ implies that

$$
\frac{1}{N^{2}} \sum_{j=1}^{N^{2}} \lambda_{j}=1
$$

So the rescaled vector of Schmidt coefficients, $\left\{\lambda_{i} / N^{2}\right\}$, can be treated as a discrete probability measure that characterizes the nonlocality of the operator $U$. To elaborate, let

$$
U \rightarrow U^{\prime}=\left(u_{A_{1}} \otimes u_{B_{1}}\right) U\left(u_{A_{2}} \otimes u_{B_{2}}\right)
$$

where $u_{A_{j}, B_{k}}$ are "local" unitary operators. In the language of dynamics, they constitute single-particle evolutions. The content of nonlocality of $U$ and $U^{\prime}$ is identical, and hence the measures characterizing their nonlocality must be the same. In the case of states, this constitutes the condition that all entanglement measures be local unitary invariants. It is clear from the definition of the operator Schmidt decomposition that the set $\left\{\lambda_{i}\right\}$ are $N^{2}$ such invariants, as $M_{A_{j}} \rightarrow u_{A_{1}} M_{A_{j}} u_{A_{2}}$ also constitute an operator basis consisting of orthonormal operators, and similarly for $M_{B_{j}}$.

Another set of $N^{2}$ invariants are constructed from the operator Schmidt decomposition of the operator product $U S$, where $S$ is the SWAP (or flip) operator defined as

$$
S\left|\phi_{A}\right\rangle\left|\phi_{B}\right\rangle=\left|\phi_{B}\right\rangle\left|\phi_{A}\right\rangle, \text { or } S\left(u_{A} \otimes u_{B}\right) S=u_{B} \otimes u_{A}
$$

for arbitrary states $\left|\phi_{A, B}\right\rangle$ and operators $u_{A, B}$. Let

$$
U S=\sum_{j=1}^{N^{2}} \sqrt{\mu_{j}} \tilde{M}_{A_{j}} \otimes \tilde{M}_{B_{j}}
$$

be its Schmidt decomposition. As $S$ is unitary, we also have that

$$
\frac{1}{N^{2}} \sum_{j=1}^{N} \mu_{j}=1
$$

That the set $\left\{\mu_{i}\right\}$ constitute $N^{2}$ invariants follows from the observation that

$$
\begin{aligned}
U^{\prime} S & =\left(u_{A_{1}} \otimes u_{B_{1}}\right) U\left(u_{A_{2}} \otimes u_{B_{2}}\right) S \\
& =\left(u_{A_{1}} \otimes u_{B_{1}}\right) U S\left(u_{B_{2}} \otimes u_{A_{2}}\right),
\end{aligned}
$$

and hence the Schmidt eigenvalues of $U S$, the $\mu_{i}$, are the same as the Schmidt eigenvalues of $U^{\prime} S$. The product $S U$ does not produce any newer invariants.

This paper is focused on these two sets of invariants and quantities derived from them. In particular, their moments and entropies provide measures of how nonlocal the operator $U$ is, leading to a class of operator entanglement entropies. Here, we will be concerned with the entropies related to the second moments, given by

$$
E(U)=1-\frac{1}{N^{4}} \sum_{j=1}^{N^{2}} \lambda_{j}^{2} \text { and } E(U S)=1-\frac{1}{N^{4}} \sum_{j=1}^{N^{2}} \mu_{j}^{2}
$$

$E(U)$ and $E(U S)$ are the linear operator entanglement entropies of the operators $U$ and $U S$, respectively. They take values in $\left[0,1-1 / N^{2}\right]$ and $E(U)=0$ iff $U$ is a local product operator. 


\section{B. Entangling power and a complementary quantity}

Notice that $E(U)$ and $E(U S)$ are in some sense complementary quantities, as for a product operator,

$E\left(u_{A} \otimes u_{B}\right)=0$, while $E\left(\left(u_{A} \otimes u_{B}\right) S\right)=E(S)=1-\frac{1}{N^{2}}$.

The last relation follows from the Schmidt decomposition of $S$, which is

$$
S=\sum_{i, k=1}^{N} e_{i k} \otimes e_{k i}, \text { where } e_{i k}=|i\rangle\langle k| .
$$

Here $\{|i\rangle, 1 \leqslant i \leqslant N\}$ denotes any orthogonal basis and hence represents a continuous family of possible Schmidt decompositions, each with $\lambda_{j}=1$ for $1 \leqslant j \leqslant N^{2}$.

The SWAP operator has the maximum operator entanglement entropy according to any measure of entropy, including the linear one, $E(S)$ as above. Thus if $E(U)=E(S)$ the operator is $U$ maximally entangled. The complementary quantity $E(U S)$ vanishes in the case of the SWAP gate, that is, $E(U S)=E\left(S^{2}\right)=0$. In fact, linear combinations of these two complementary quantities give rise to two measures that are extensively discussed in this paper.

One of the two measures we look at is the well-studied "entangling power." The entangling power $e_{p}(U)[9,10]$ of an operator $U \in \mathcal{H}_{A}^{N} \otimes \mathcal{H}_{B}^{N}$ is defined as the average entanglement created when $U$ acts on the product state $\left|\psi_{A}\right\rangle\left|\psi_{B}\right\rangle$ sampled according to the Haar measure on the individual spaces:

$$
e_{p}(U)=\left(\frac{N+1}{N-1}\right) \overline{\mathcal{E}\left(U\left|\psi_{A}\right\rangle\left|\psi_{B}\right\rangle\right)} \psi_{A}, \psi_{B}
$$

Here the entanglement measure is the linear entropy $\mathcal{E}(|\psi\rangle)=$ $1-\operatorname{tr}_{A}\left(\rho_{A}^{2}\right)$, and $\rho_{A}$ is the reduced density matrix $\operatorname{tr}_{B}(|\psi\rangle\langle\psi|)$. It has been shown in [10] that for any gate $U$ its entangling power can be expressed by the linear operator entanglement entropy,

$$
e_{p}(U)=\frac{1}{E(S)}[E(U)+E(U S)-E(S)] .
$$

The range of $e_{p}(U)$ is

$$
0 \leqslant e_{p}(U) \leqslant 1,
$$

which follows from the fact that the maximum value of $E(U)$ is $E(S)$. We have rescaled the definition of $e_{p}(U)$ from that originally defined in [9] so that the maximum value is simply 1 independent of $N$.

If $e_{p}(U)=0$, then $U$ is either a product of local operators or it is locally equivalent to the SWAP. The fact that SWAP does not create any entanglement when acting on product states leads to $e_{p}(S)=0$, but that it is highly nonlocal is reflected in its operator entanglement being maximum. This is one motivation for introducing the complementary quantity

$$
g_{t}(U):=\frac{1}{2 E(S)}[E(U)-E(U S)+E(S)],
$$

where $g_{t}$ is referred to as gate typicality in [44]. The range of $g_{t}(U)$ is

$$
0 \leqslant g_{t}(U) \leqslant 1,
$$

and $g_{t}(U)=1$ iff $U$ is the SWAP or is locally equivalent to the SWAP. Again, we have rescaled $g_{t}$ from the original definition in [44] by a factor of 2 for complete parity with $e_{p}$.

Thus while $e_{p}$ does not distinguish the local operators from the SWAP, $g_{t}$ does. It turns out that rather than discussing the pair $\{E(U), E(U S)\}$ in several settings, it seems more natural to work in the plane $\left\{e_{p}(U), g_{t}(U)\right\}$. The average of these measures when $U$ is sampled uniformly from the space of unitary matrices with respect to the Haar measure constitutes the average over the circular unitary ensemble (CUE), and it reads

$$
\bar{E}=\overline{e_{p}}=\frac{N^{2}-1}{N^{2}+1}=\frac{E(S)}{2-E(S)}, \quad \overline{g_{t}}=\frac{1}{2} .
$$

As the scale is set so that $g_{t} \in[0,1]$, and the Haar average reads $1 / 2$, we see that both classes of local gates and gates locally equivalent to SWAP are equally nontypical. The fact that $\bar{E}$ and $\overline{e_{p}}$ are close to the maximal possible value indicates that a typical Haar unitary gate has strong entangling properties [49], in analogy to the known fact that a generic bipartite pure state is strongly entangled [15,50].

Computation of the operator Schmidt decomposition and the operator entanglements follows from suitable permutations of the unitary matrix. If $\langle i \alpha|U| j \beta\rangle=\left\langle i j\left|U^{R}\right| \alpha \beta\right\rangle$ and $\langle i \alpha|U| j \beta\rangle=\left\langle j \alpha\left|U^{T_{A}}\right| i \beta\right\rangle$ denote the reshuffling (also referred to as realignment) and the partial transpose operations, respectively, we may define the following density matrices [12]:

$$
\rho_{R}(U)=\frac{1}{N^{2}} U^{R} U^{R \dagger}, \quad \rho_{T}(U)=\frac{1}{N^{2}} U^{T_{A}} U^{T_{A} \dagger} .
$$

Their linear entropies are given by

$$
E(U)=1-\frac{1}{N^{4}} \operatorname{tr}\left(U^{R} U^{R \dagger}\right)^{2}
$$

and

$$
E(U S)=1-\frac{1}{N^{4}} \operatorname{tr}\left(U^{T_{A}} U^{T_{A} \dagger}\right)^{2} .
$$

The operational interpretations of these quantities in terms of state entanglement of the equivalent four-party system are elaborated on in Appendix A, including the generalization to the case of unequal subsystem dimensionality.

Note that if $U^{R}$ is also unitary, then $E(U)=E(S)$ is the maximum possible.

Unitary operators whose reshuffling is also unitary have recently been called dual-unitaries due to their appearance in lattice models with space-time duality [28,51,52]. This class contains, for instance, the discrete Fourier transform $F_{N^{2}}$, for which all coefficients in the operator Schmidt decomposition are equal $[49,53]$. This dual-unitary property allows for special many-body systems built out of such unitaries to be solvable in some sense [54-56], although they can be nonintegrable. It is indeed interesting that such unitaries are also maximally entangled in the operator entanglement sense. A way to generate ensembles of dual unitaries has been presented in [57]. 


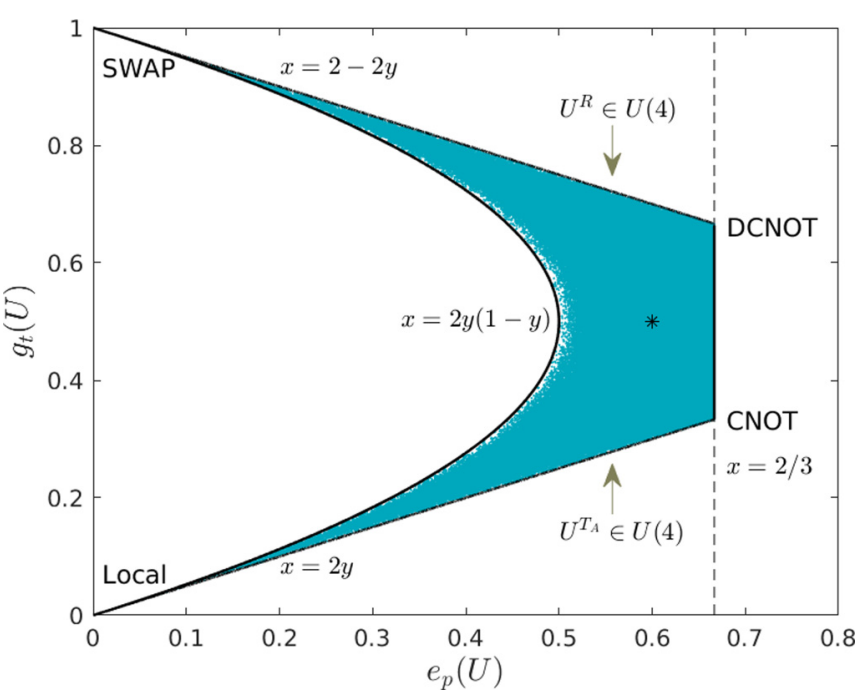

FIG. 1. Set $K_{2}$ of all two-qubit gates projected into the plane entangling power, $x=e_{p}(U)$, vs gate-typicality, $y=g_{t}(U)$. Each point corresponds to a random unitary matrix from $U(4)$, while (*) represents the average over CUE. The boundaries $\partial K_{2}$ are identified in the text and in Fig. 2.

If $U^{T_{A}}$ is also unitary, apart from $U^{R}$, then $E(U S)=E(S)$. Such a matrix $U$, called "2-unitary" [58], saturates the maximum of entangling power, set to unity by our normalization. As discussed in Appendix A, any two-unitary matrix of order $N^{2}$ corresponds to a two-uniform state of four quNits, maximally entangled with respect to three possible symmetric partitions of the system [59]. Any dual-unitary, which satisfies weaker constraints, represents a four-party state maximally entangled with respect to two possible partitions out of three. Any unitary matrix of size $N^{3}$, which remains unitary for any possible choice of three indices out of six, is called threeunitary. It maximizes the tripartite entangling power [60], and it corresponds to a three-uniform state of six parties, maximally entangled with respect to any splitting of the system into three plus three parts. In general, a $k$-unitary matrix of size $N^{k}$ represents a $k$-uniform pure state of $2 k$ subsystems [58], maximally entangled with respect to any symmetric partition of the system, and therefore called an absolutely maximally entangled (AME) state.

\section{BOUNDARIES OF TWO-QUBIT GATES}

We focus on the two simple cases of two-qubit and twoqutrit unitary gates. In particular, for $N=2$ and 3 we study the structure of the set $K_{N}$ of unitary matrices, $U\left(N^{2}\right)$, projected onto the plane $\left\{e_{p}(U), g_{t}(U)\right\}$. Due to the normalization used, the phase space is restricted to the square $[0,1]^{2}$. We will be interested in describing the boundary of the allowed area within the square and identifying particular gates corresponding to the distinguished points of the boundary.

The gate typicality $g_{t}$ and entangling power $e_{p}$ for twoqubit unitaries $U$, drawn at random from CUE(4), are shown in Fig. 1. It is clear that $0 \leqslant e_{p} \leqslant 2 / 3$, reflecting the wellknown fact that the maximum possible value of entangling power for a two-qubit gate is not 1 (with our choice of factors), rather it is only $2 / 3$ [9]. This is related to the nonexistence of absolutely maximally entangled states for a four-qubit system [46], as already mentioned above and explained in Appendix A.

Gate typicality is symmetric about its mean value ${\overline{g_{t}(U)}}^{U}=1 / 2$, and this is reflected by the following equality:

$$
g_{t}(U)+g_{t}(U S)=1 .
$$

Its maximal value $g_{t}=1$ is attained only by the SWAP gate and its local equivalents, while the minimal value $g_{t}=0$ corresponds to local operators. Therefore, it might be appropriate to call the operators with $1 / 2 \leqslant g_{t} \leqslant 1$ "swAP-like."

The boundaries of the set $K_{2}$ shown in Fig. 1 can be found using the limits of operator entanglement $E(U)$ and $E(U S)$. Writing these quantities in terms of the entangling power $e_{p}$ and gate typicality $g_{t}$ of a two-qubit operator, $N=2$, leads to

$$
\begin{aligned}
E(U) & =\frac{3}{8}\left[e_{p}(U)+2 g_{t}(U)\right], \\
E(U S) & =\frac{3}{8}\left[e_{p}(U)-2 g_{t}(U)+2\right] .
\end{aligned}
$$

The upper bounds on $E(U)$ and $E(U S)$ (equal to 3/4) lead to the relations

$$
e_{p}+2 g_{t} \leqslant 2 \text { and } g_{t} \geqslant e_{p} / 2,
$$

which are the top and bottom lines in Fig. 1. The maximum value of $e_{p}=2 / 3$ is reached by the CNOT gate and is an "optimal" gate in the terminology of [9]. The region is further restricted, however, and we will show below that the left boundary is given by the parabola $e_{p}=2 g_{t}\left(1-g_{t}\right)$. We further show in Sec. IV that this boundary in fact consists of gates of the form $S^{\alpha}$ with $0 \leqslant \alpha \leqslant 1$, which are rational powers of the SWAP operator $S$.

\section{The Weyl chamber and various gates}

While the lines in Eq. (26) are bounds, we identify the gates that make these actual boundaries of the allowed set $K_{2}$ in the space $e_{p}$ versus $g_{t}$. It will be useful to work with the well known canonical form of a two-qubit unitary operator. Any two-qubit operator $U \in S U$ (4) [61], up to left and right multiplication by local unitaries, can be expressed in terms of Euler angles $\left\{c_{1}, c_{2}, c_{3}\right\} \in[0, \pi]$ as [62-65]

$$
U=\exp \left[-i\left(\frac{c_{1}}{2} \sigma_{1} \otimes \sigma_{1}+\frac{c_{2}}{2} \sigma_{2} \otimes \sigma_{2}+\frac{c_{3}}{2} \sigma_{3} \otimes \sigma_{3}\right)\right],
$$

where $\left\{\sigma_{1}, \sigma_{2}, \sigma_{3}\right\}$ are the Pauli matrices. In the standard computational basis (the eigenbasis of $\sigma_{3}$ ), any bipartite unitary operator can thus be written as

$$
U=\left(\begin{array}{cccc}
e^{\frac{-i c_{3}}{2}} c^{-} & 0 & 0 & -i e^{\frac{-i c_{3}}{2}} s^{-} \\
0 & e^{\frac{i c_{3}}{2}} c^{+} & -i e^{\frac{i c_{3}}{2}} s^{+} & 0 \\
0 & -i e^{\frac{i c_{3}}{2}} s^{+} & e^{\frac{i c_{3}}{2}} c^{+} & 0 \\
-i e^{\frac{-i c_{3}}{2}} s^{-} & 0 & 0 & e^{\frac{-i c_{3}}{2}} c^{-}
\end{array}\right),
$$

where

$$
c^{ \pm}=\cos \left[\left(c_{1} \pm c_{2}\right) / 2\right], \quad s^{ \pm}=\sin \left[\left(c_{1} \pm c_{2}\right) / 2\right] .
$$

On imposing the constraint of local unitary equivalence, that is, if any two unitaries $U$ and $U^{\prime}=\left(u_{A_{1}} \otimes u_{B_{1}}\right) U\left(u_{A_{2}} \otimes u_{B_{2}}\right)$ related by local unitaries are represented by the same set of 
Euler angles, the range of values gets restricted to $\left|c_{3}\right|<c_{2}<$ $c_{1}<\pi / 2$. This region in the $\left\{c_{1}, c_{2}, c_{3}\right\}$ space containing the nonlocal two-qubit gates forms a tetrahedron known as the Weyl chamber [64].

In terms of the $\left\{c_{1}, c_{2}, c_{3}\right\}$ parametrization, it is known $[64,66]$ that one can define two quantities that are invariant under local unitary operations, namely

$$
\begin{aligned}
G_{1}= & \cos ^{2} c_{1} \cos ^{2} c_{2} \cos ^{2} c_{3}-\sin ^{2} c_{1} \sin ^{2} c_{2} \sin ^{2} c_{3} \\
& +\frac{i}{4} \sin 2 c_{1} \sin 2 c_{2} \sin 2 c_{3}, \\
G_{2}= & \cos 2 c_{1}+\cos 2 c_{2}+\cos 2 c_{3} .
\end{aligned}
$$

The operator entanglements $E(U)$ and $E(U S)$ can be written in terms of local invariants $G_{1}$ and $G_{2}$ as follows [67]:

$$
\begin{aligned}
E(U) & =1-\frac{1}{8}\left[3+2\left|G_{1}(U)\right|+G_{2}(U)\right], \\
E(U S) & =1-\frac{1}{8}\left[3+2\left|G_{1}(U)\right|-G_{2}(U)\right] .
\end{aligned}
$$

Consequently, the entangling power and gate-typicality of any two-qubit gate $U$ can be explicitly evaluated in terms of the angles $\left\{c_{1}, c_{2}, c_{3}\right\}$, and it takes on an elegant and simple form as

$$
\begin{aligned}
& e_{p}(U)=\frac{2}{3}\left[\sin ^{2} c_{1} \cos ^{2} c_{2}+\sin ^{2} c_{2} \cos ^{2} c_{3}+\sin ^{2} c_{3} \cos ^{2} c_{1}\right], \\
& g_{t}(U)=\frac{1}{3}\left[\sin ^{2} c_{1}+\sin ^{2} c_{2}+\sin ^{2} c_{3}\right] .
\end{aligned}
$$

This leads to the following restriction on the allowed region in the $e_{p}-g_{t}$ plane for two-qubit gates.

Theorem III.1: Boundary of two-qubit gates. The entangling power $e_{p}(U)$ and gate-typicality $g_{t}(U)$ for any two-qubit unitary $U$ satisfy

$$
e_{p}(U) \geqslant 2 g_{t}(U)\left[1-g_{t}(U)\right] .
$$

Proof: Using Eq. (31), we see that $2 g_{t}(U)\left[1-g_{t}(U)\right]$ is of the form

$$
2 g_{t}(U)\left[1-g_{t}(U)\right]=\frac{2}{9}(x+y+z)[3-(x+y+z)],
$$

where $x \equiv \sin ^{2} c_{1}, y \equiv \sin ^{2} c_{2}$, and $z \equiv \sin ^{2} c_{3}$ satisfy $0 \leqslant$ $x, y, z \leqslant 1$. Then, it is easy to see that

$$
\begin{aligned}
(x+ & y+z)[3-(x+y+z)] \\
= & 3[x(1-y)+y(1-z)+z(1-x)] \\
& +(x y+y z+z x)-\left(x^{2}+y^{2}+z^{2}\right) \\
\leqslant & 3[x(1-y)+y(1-z)+z(1-x)],
\end{aligned}
$$

since $x y+y z+z x \leqslant x^{2}+y^{2}+z^{2}$, by the Schwarz inequality. Using this in Eq. (33) above, we get

$$
\begin{aligned}
& 2 g_{t}(U)\left[1-g_{t}(U)\right] \\
& \quad \leqslant \frac{2}{3}[x(1-y)+y(1-z)+z(1-x)] \\
& \quad=\frac{2}{3}\left(\sin ^{2} c_{1} \cos ^{2} c_{2}+\sin ^{2} c_{2} \cos ^{2} c_{3}+\sin ^{2} c_{3} \cos ^{2} c_{1}\right) \\
& \quad=e_{p}(U)
\end{aligned}
$$

as desired.

The inequality in Eq. (32) is tight, as the $S^{\alpha}$ family of gates with $0 \leqslant \alpha \leqslant 1$ lie on the parabola $e_{p}(U)=$ $2 g_{t}(U)\left[1-g_{t}(U)\right]$. This is shown by an explicit calculation in Eq. (42), Sec. IV.

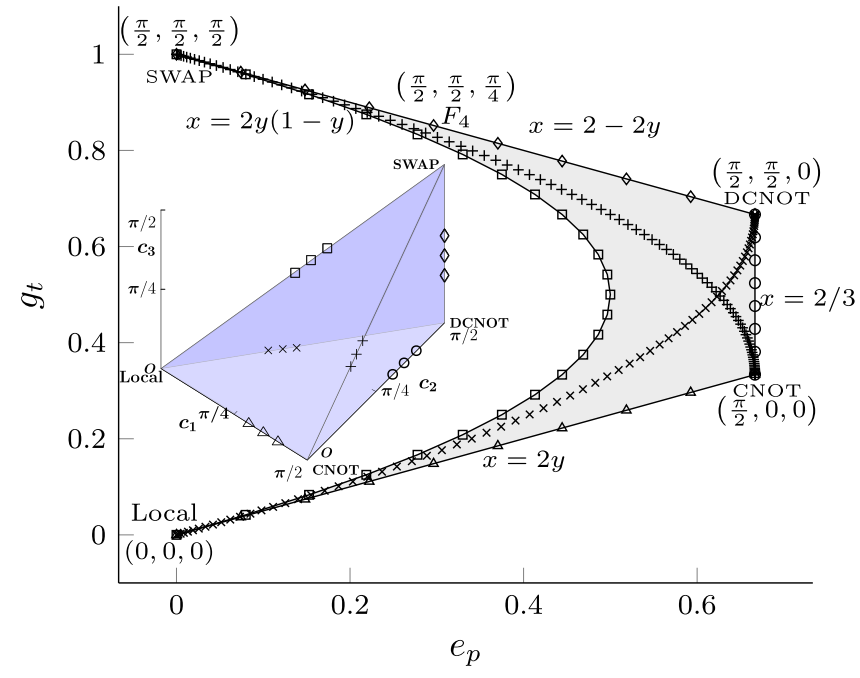

FIG. 2. Boundaries of the set $K_{2}$ representing two-qubit gates in the plane $\left(e_{p}, g_{t}\right)$ are indicated by solid lines. Note distinguished gates identified in the plot. The inset shows edges of the tetrahedron in the parameter space $\left(c_{1}, c_{2}, c_{3}\right)$ forming half of the Weyl chamber [45], which corresponds to $\partial K_{2}$.

The CNOT gate $C$ has the maximum entangling power of $2 / 3$, as expected. Furthermore, we show below that all members of the $C S^{\alpha}$ family, with $0 \leqslant \alpha \leqslant 1$, have maximum entangling power of $2 / 3$ and form the rightmost vertical boundary in Figs. 1 and 2. The gate $C S$ is the so-called doubleCNOT (DCNOT) gate [68]. Note that

$$
U_{t}=\exp (i t S)=\mathbb{1} \cos t+i \sin t S
$$

as $S^{2}=\mathbb{1}$, where $\mathbb{1}$ denotes the identity operator. This is a route to defining fractional powers of $S$ as $\exp (i \pi S / 2)=i S$, and therefore $(i S)^{t 2 / \pi}$ is the same as $\exp (i t S)$, and the overall phase of $i^{t 2 / \pi}$ makes no difference to any of the subsequent calculations. Therefore, $U_{t}$ is essentially $S^{2 t / \pi}$. The reshuffled matrix of $C S^{\alpha}$ up to a constant phase is given by

$$
\left(C S^{\alpha}\right)^{R}=\cos (\pi \alpha / 2) C^{R}+i \sin (\pi \alpha / 2)(C S)^{R} .
$$

The rearrangement of the CNOT gate is nonunitary, being $|00\rangle\langle 00|+| 00\rangle\langle 11|+| 11\rangle\langle 01|+| 11\rangle\langle 10|$, while $(C S)^{R}$ is again a permutation given by $|00\rangle\langle 00|+| 10\rangle\langle 11|+$ $|01\rangle\langle 10|+| 11\rangle\langle 01|$. A calculation then yields

$$
E\left(C S^{\alpha}\right)=\frac{1}{8}[5-\cos (\pi \alpha)] .
$$

Hence

$$
e_{p}\left(C S^{\alpha}\right)=\frac{1}{E(S)}\left[E\left(C S^{\alpha}\right)+E\left(C S^{\alpha+1}\right)-E(S)\right]=\frac{2}{3},
$$

and $g_{t}\left(C S^{\alpha}\right)=1 / 2-\cos (\pi \alpha) / 6$ interpolating between $1 / 3$ and $2 / 3$.

Several other standard two-qubit gates are identified, and their operator entanglement and entangling powers are given in Table I. We also identify gates in the Weyl chamber with different regions of the set $K_{2}$ contained in the plane $\left(e_{p}, g_{t}\right)$. In Fig. 2, six edges of the tetrahedron forming a half of the chamber [45] are shown. Four of these edges form four of the boundaries $\partial K_{2}$; the other two connect two of the extreme points symmetrically. 
TABLE I. Nonlocal properties of selected two-qubit gates, $N=$ 2. Their location in the set $K_{2}$ is shown in Fig. 2 .

\begin{tabular}{lcccc}
\hline \hline Gate $U$ & $E(U)$ & $E(U S)$ & $e_{p}(U)$ & $g_{t}(U)$ \\
\hline Local-gate & 0 & $\frac{3}{4}$ & 0 & 0 \\
$\sqrt{\text { CNOT }}$ & $\frac{1}{4}$ & $\frac{3}{4}$ & $\frac{1}{3}$ & $\frac{1}{6}$ \\
CNOT, B-GATE & $\frac{1}{2}$ & $\frac{3}{4}$ & $\frac{2}{3}$ & $\frac{1}{3}$ \\
DCNOT & $\frac{3}{4}$ & $\frac{1}{2}$ & $\frac{2}{3}$ & $\frac{2}{3}$ \\
Fourier $F_{4}$ & $\frac{3}{4}$ & $\frac{1}{4}$ & $\frac{1}{3}$ & $\frac{5}{6}$ \\
$\sqrt{\text { SWAP }}$ & $\frac{9}{16}$ & $\frac{9}{16}$ & $\frac{1}{2}$ & $\frac{1}{2}$ \\
SWAP & $\frac{3}{4}$ & 0 & 0 & 1 \\
Haar average & $\frac{3}{5}$ & $\frac{3}{5}$ & $\frac{3}{5}$ & $\frac{1}{2}$ \\
\hline \hline
\end{tabular}

\section{BEYOND QUBITS AND THE ENTANGLING POWER OF SOME QUNIT GATES}

Moving beyond qubits, we now study the entanglement landscape of bipartite unitary gates acting in a composite $N \times N$ quantum system. In this context, we investigate the Fourier gate and the fractional powers of the SWAP that form an important family of gates. We observe that for any $N \geqslant 2$, the fractional powers of SWAP lie on a parabola. The rightmost point is maximally entangling, at $e_{p}=1$ and $g_{t}=1 / 2$, and it is known that in all dimensions except $N=6$ (and $N=2$, which we have already dealt with) permutations exist that have these values. In the case $N=3$, explicit examples of permutations that have $e_{p}=1$ have been constructed $[58,69]$.

The discrete Fourier transform (DFT) on the space $\mathcal{H}_{N} \otimes$ $\mathcal{H}_{N}$ is given by the unitary gate $F_{N^{2}}$ of order $N^{2}$, with entries $F_{m n}=\frac{1}{N} \exp \left(2 \pi i m n / N^{2}\right)$. This may be expressed in bipartite notation as

$$
\left\langle k \alpha\left|F_{N^{2}}\right| j \beta\right\rangle=\frac{1}{N} e^{\frac{2 \pi i}{N^{2}}[(k+\alpha N)(j+\beta N)],}
$$

where $0 \leqslant k, j, \alpha, \beta \leqslant N-1$. It is then straightforward to verify that the reshuffled matrix $F_{N^{2}}^{R}$ is also unitary [49], and hence the operator entanglement attains the maximum possible value, namely, that is most possible is $E\left(F_{N^{2}}\right)=1-1 / N^{2}$. In this sense, the Fourier gate in arbitrary dimensions is a dual-unitary, and a recent paper [55] constructs dual kicked chains using the DFT to study solvable Floquet many-body systems.

However, the partial transpose of the DFT is not unitary, and hence the Fourier gate does not have maximal entangling power. Equivalently, $E\left(F_{N^{2}} S\right)$ is not the most possible; instead, a calculation yields

$$
\begin{aligned}
E\left(F_{N^{2}} S\right)= & 1-\frac{1}{N^{4}}\left[N^{3}+2 \sum_{k=1}^{N-1} k \frac{\sin ^{2}(k \pi / N)}{\sin ^{2}\left(\pi / N-k \pi / N^{2}\right)}\right] \\
& \approx 1-\frac{2}{\pi^{2}} \int_{0}^{1} \frac{x \sin ^{2}(\pi x)}{(1-x)^{2}} d x \approx 0.344
\end{aligned}
$$

where the approximation is valid for large $N$. Thus the operator entanglement of $F_{N^{2}} S$ and the entangling power of the Fourier gate $F_{N^{2}}$ tends to $\approx 0.344$, about one-third of the maximum possible value.

As indicated in Eq. (35) above, the fractional powers of the SWAP $S$ up to phase factors are given by $U_{t}=\exp (i t S)$. Since the reshuffled operator $S^{R}=S$, we get

$$
U_{t}^{R}=\mathbb{1}^{R} \cos t+i \sin t S=N\left|\Phi^{+}\right\rangle\left\langle\Phi^{+}\right| \cos t+i \sin t S,
$$

where we have used the fact that the reshuffling of the identity is given by $\mathbb{1}^{R}=N\left|\Phi^{+}\right\rangle\left\langle\Phi^{+}\right|$, with $\left|\Phi^{+}\right\rangle=\frac{1}{\sqrt{N}} \sum_{i=1}^{N}|i i\rangle$ being a maximally entangled state. Further, as $U_{t} S=S \cos t+$ $i \mathbb{1} \sin t$, the following simple formulas follow for the fractional powers of the SWAP gate:

$$
\begin{aligned}
& E\left(e^{i t S}\right)=E(S)\left(1-\cos ^{4} t\right), E\left(e^{i t S} S\right)=E(S)\left(1-\sin ^{4} t\right), \\
& e_{p}\left(e^{i t S}\right)=\frac{1}{2} \sin ^{2}(2 t), g_{t}\left(e^{i t S}\right)=\sin ^{2} t .
\end{aligned}
$$

Thus, if $U_{t}$ is a fractional power of $S$, then $e_{p}\left(U_{t}\right)=$ $2 g_{t}\left(U_{t}\right)\left[1-g_{t}\left(U_{t}\right)\right]$ in any dimension. We have already shown that this parabola is indeed the left-boundary of the set $K_{2}$ in the $\left(e_{p}, g_{t}\right)$ plane in the case of two-qubit gates.

To investigate the neighborhood of the parabola, we start with an operator of the form $S^{\alpha}$ and perturb it while retaining the unitarity. There are many possible ways of doing such a perturbation, all of which yield equivalent results. For example, one may deform $S^{\alpha} \rightarrow S^{\alpha} \exp (i \epsilon H)$, where $H$ is a random Hermitian matrix with unit variance and zero mean elements. Another approach is to use random matrices $V_{\epsilon}=U_{\mathrm{CUE}} U_{d}(\epsilon) U_{\mathrm{CUE}}^{\dagger}$ from the ensemble investigated in [70] and defined by a Haar random unitary matrix $U_{\text {CUE }}$ and a diagonal matrix $U_{d}(\epsilon)$ with phases $\exp (i \epsilon \xi)$, where $\xi$ is a uniform random number in $[-\pi, \pi)$. Powers of SWAP perturbed as $S^{\alpha} \rightarrow S^{\alpha} V_{\epsilon}$ result in values of $\left\{e_{p}, g_{t}\right\}$ lying to the right of the parabola. Combined with the stationarity derived in Appendix B, one may be tempted to conjecture that the parabola itself is a boundary. However, we have found an exception in a permutation in the qutrit case, and we can only conclude that typical perturbations of $S^{\alpha}$ result in a movement to the right of the parabola in the $\left(e_{p}, g_{t}\right)$ plane.

A similar study to that in the case of $K_{2}$ was performed for unitary matrices belonging to the lower and the upper parts of the boundary of the set $K_{3}$. It is useful to distinguish certain unitary matrices, which correspond to points at $\partial K_{3}$. The controlled addition gate $C_{N}$ acting on a two-quNit system can be considered as a generalization of the standard CNOT gate. In the case of $N=3$, such a gate reads

$$
C_{3}|i\rangle \otimes|j\rangle=|i\rangle \otimes|i \oplus j\rangle, i, j \in \mathbb{Z}_{3},
$$

where $\oplus$ denotes addition modulo 3 . This gate attains the maximal value of $E\left(C_{3} S\right)$ and lies in $K_{3}$ on its lower boundary, $y=2 x$. It is seen that the perturbations have the tendency to quickly approach the CUE "cloud" in the manner of a jet.

In Fig. 3, the neighborhood gates of several unitary quantum gates are generated for $N=3$, and the corresponding phase-space plot is shown. The rightmost point of the set $K_{3}$ in the $\left(e_{p}, g_{t}\right)$ plane, denoted as $P_{9}$ in Fig. 3 , corresponds to one of the permutations with $e_{p}=1$ defined in $[58,69]$. The Fourier matrix $F_{9}$ attains the maximum value of $E(U)$, as $F_{9}^{R}$ is unitary, and it lies on the upper boundary of $K_{3}$ formed by the line $x=2(1-y)$.

The upper boundary line contains maximally entangled unitary matrices for which $U^{R}$ is also unitary. However, the partially transposed matrix $U^{T_{a}}$ is not unitary, with the 


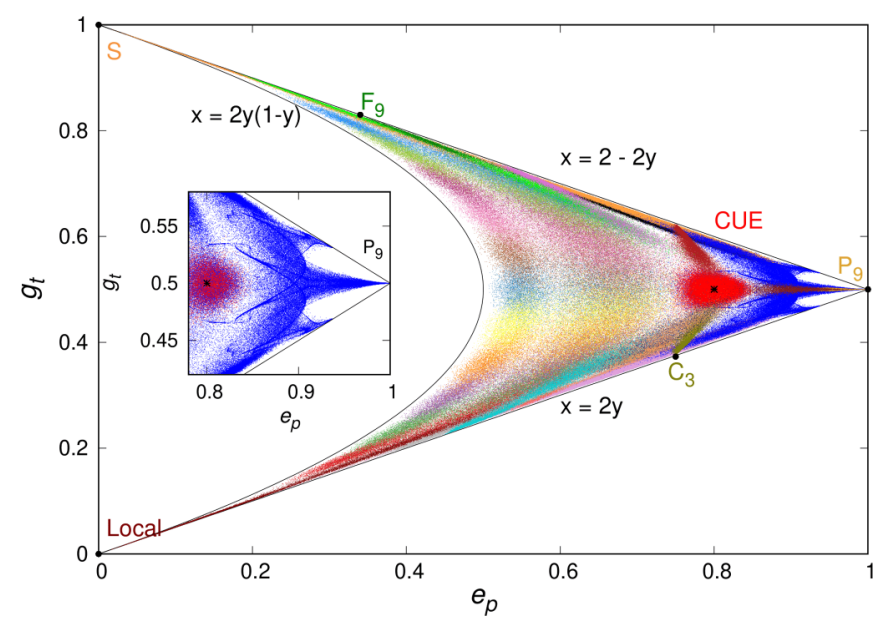

FIG. 3. Unitary matrices $U \in U(9)$, representing two-qutrit gates, projected into the set $K_{3}$ in the plane $\left(e_{p}, g_{t}\right)$. Each color represents the neighborhood of a particular gate, labeled with the same color. The upper side of the triangle, including Fourier matrix $F_{9}$, contains "dual unitaries" for which $U^{R}$ is unitary, while the lower side includes these for which $U^{T_{A}}$ is unitary. The inset provides a magnified view of the region around the rightmost point representing the 2-unitary gate $P_{9}$, with the CUE "cloud," centered at $(1 / 2,4 / 5)$ $(*)$, and shown in red, and perturbations of different boundary gates in blue.

exception of the matrices at the right corner of the triangle. Thus gates belonging to the upper boundary of $K_{N}$ are not 2-unitary [58], but they satisfy the weaker condition of being dual unitaries [52]. Unitary gates for which $U^{T_{A}}$ is unitary, studied in $[71,72]$ in the context of quantum operations preserving some given matrix algebra, belong to the lower boundary line of $K_{3}$. Both lines cross at the right corner of the triangle, representing permutation $P_{9}$ and other 2-unitary matrices, which maximize the entangling power.

It is interesting to observe that the set $K_{3}$ seems not to fill the entire edge of the triangle close to the corner with $e_{p}=1$, as no dual unitaries in the vicinity of $P_{9}$ were found. This fact is borne out by numerical simulations that employ an algorithm to create an ensemble of dual ones [57]. The significance of the gap observed is to be fully explored, but the numerics suggest that the set of dual unitary matrices of size $N^{2}=9$ is not connected, in contrast to the two-qubit case, $N^{2}=4$. Since the dual unitary operators are related to four-party entanglement-see Appendix A-this implies some additional constraints on the entanglement in four-qutrit systems across different partitions and on possible spectra of two-partite density matrices obtained by partial trace of a pure state of size $N^{4}$.

Analysis of the nonlocal properties of any two-qubit gate becomes easier as the canonical form (27) is valid for any unitary matrix from $U(4)$. This form, related to isomorphism in group theory between $S O(4)$ and $S U(2) \times S U(2)$, cannot be generalized for two-qutrit gates. Therefore, our understanding of the set of bipartite gates acting on $N \times N$ systems is still not complete. The structure of the set $K_{N}$ obtained by a projection of $U\left(N^{2}\right)$ onto the plane $\left(e_{p}, g_{t}\right)$ is not entirely characterized even in the case $N=3$. Leaving these open problems for further studies, we shall now move to a related problem, namely if a given bipartite unitary gate $U$ acts sequentially on a quantum system.

\section{TIME EVOLUTION AND MULTIPLE USES OF THE NONLOCAL OPERATORS}

If $U$ is a bipartite quantum propagator, it is natural to consider a combination $\mathcal{U}=\left(u_{A} \otimes u_{B}\right) U$ where the unitaries $u_{A}, u_{B}$ are interpreted as "local dynamics" or single-particle dynamics. We have motivated [see the discussion around Eq. (4)] the study of its powers $\mathcal{U}^{n}$ as well as products $\mathcal{U}^{(n)}$ with different local operators in each term of the product.

The circuit in Fig. 4 describes the time-evolution scenario considered here for the case of qubit systems. Specifically, the circuit depicts the propagator $\mathcal{U}^{(n)}$ for $n=3$. The fixed nonlocal unitary $U \in U(4)$ is implemented via a combination of CNOT gates and local rotations $R_{z}(t)$ and $R_{y}(t)$, following the prescription in [73]. The interlacing local qubit gates are denoted as $A_{i}$ and $A_{i}^{\prime}$, with $i=1,2$. We have omitted the initial set of local unitaries since they do not affect the entangling power. Note that the interlacing locals are different at each step, and hence labeled differently.

Observe that for a single time step, the nonlocal content of $U$ is the same as that of $\mathcal{U}$, hence $e_{p}(U)=e_{p}(\mathcal{U})$. Thus if the gate $\mathcal{U}$ is applied onto an unentangled initial state, the local dynamics does not play any role in the creation of quantum entanglement. However, the nonlocal content of multiple applications, either as $\mathcal{U}^{n}$ or $\mathcal{U}^{(n)}$, which represents discrete time evolution, is a different matter as the Schmidt coefficients of an operator in general change on taking powers. In this case, the local dynamics can play a crucial role $[44,45]$. For instance, in terms of entangling power,

$$
e_{p}\left(\mathcal{U}^{2}\right)=e_{p}\left[U\left(u_{A} \otimes u_{B}\right) U\right] \neq e_{p}\left(U^{2}\right) .
$$

One of the aims of this paper is to analyze this difference and study the regime of large $n$. While we presented related results earlier [44], this work contains an important generalization and a more elegant derivation that uses group theory. Note that we are interested in generic statements about average entanglement growth in time, a subject that already has a considerable literature and is still a topic of research.

\section{A. Thermalization of entangling power}

The generalization allows for the subsystems $\mathcal{H}^{A}$ and $\mathcal{H}^{B}$ to have different dimensions $N$ and $M$, say $N \leqslant M$. The operator entanglement still follows from the Schmidt decomposition of $U$ as in Eq. (5) and is determined by the singular values of the reshuffled matrix $U^{R}$ of size $N^{2} \times M^{2}$. This gives the vector of local invariants $\lambda_{j}$, equal to eigenvalues of a positive matrix $U^{R}\left(U^{R}\right)^{\dagger}$. The other set of invariants, which in the symmetric case came from the Schmidt decomposition of $U S$ in Eq. (9), now come from the singular values of the square matrix $U^{T_{A}}$ of size $N M \times N M$. The generalization of the expressions in (16) and (18) for entangling power [11] and gate-typicality, respectively, based on the reshuffled and partially transposed 


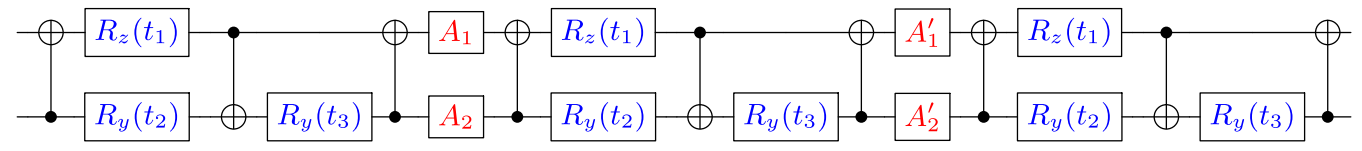

FIG. 4. Circuit representing the propagator $\mathcal{U}^{(3)}$ for a pair of qubits. The fixed nonlocal two-qubit gate $U$ is implemented via the sequence of CNOTs and rotation gates $R_{z}(t), R_{y}(t)$. The gates $A_{i}$ and $A_{i}^{\prime}$ represent the interlacing local unitaries, which vary at each time step.

matrix $U$, is given by

$$
\begin{aligned}
e_{p}(U)= & \frac{1}{M^{2}\left(N^{2}-1\right)}\{N M(N M+1) \\
& \left.-\operatorname{tr}\left[U^{R}\left(U^{R}\right)^{\dagger}\right]^{2}-\operatorname{tr}\left[U^{T_{A}}\left(U^{T_{A}}\right)^{\dagger}\right]^{2}\right\}, \\
g_{t}(U)= & \frac{1}{2 N M(N+1)(M-1)}\left\{N^{2} M^{2}-N M\right. \\
& \left.-\operatorname{tr}\left[U^{R}\left(U^{R}\right)^{\dagger}\right]^{2}+\operatorname{tr}\left[U^{T_{A}}\left(U^{T_{A}}\right)^{\dagger}\right]^{2}\right\} .
\end{aligned}
$$

Note that we use a normalization factor that implies that the maximal entangling power is equal to unity, which is attained when $\operatorname{tr}\left[U^{R}\left(U^{R}\right)^{\dagger}\right]^{2}=M^{2}$ and $\operatorname{tr}\left[U^{T_{A}}\left(U^{T_{A}}\right)^{\dagger}\right]^{2}=N M$. Hence our expression differs from the expression in [11] by a factor $\tilde{e}_{p}^{\max }=\frac{M(N-1)}{N(M+1)}$, which is the unscaled maximum entangling power for an $N \times M$ bipartite system.

The generalization in Eq. (45) allows us to consider a situation in which the bipartite interaction is nonzero but arbitrarily small and the second subsystem is considerably large, such as a thermal bath. In particular, we show in Theorem V.1 below that

$$
\begin{aligned}
\left\langle e_{p}\left[U\left(u_{A} \otimes u_{B}\right) V\right]\right\rangle_{u_{A}, u_{B}} \\
=e_{p}(U)+e_{p}(V)-e_{p}(U) e_{p}(V) / \overline{e_{p}}, \\
\quad\left\langle g_{t}\left[U\left(u_{A} \otimes u_{B}\right) V\right]\right\rangle_{u_{A}, u_{B}} \\
=g_{t}(U)+g_{t}(V)-g_{t}(U) g_{t}(V) / \overline{g_{t}} .
\end{aligned}
$$

Here $U$ and $V$ are any two unitary operators, and the angular brackets indicate averaging over the local unitary operations with $u_{A, B}$ sampled uniformly (Haar measure). The quantities $\overline{e_{p}}$ and $\overline{g_{t}}$ are the Haar averages over the $M N$-dimensional space that generalize the expressions in Eq. (20), for subsystems of equal dimensions, to

$$
\overline{e_{p}}=\frac{N\left(M^{2}-1\right)}{M(N M+1)}, \quad \overline{g_{t}}=\frac{(N-1)(M+1)}{2(N M-1)} .
$$

The above discussion can be directly related to operator scrambling, which measures the spread of an initially localized operator $[2,7,8,33]$.

In the simplest bipartite setting, operator scrambling can be characterized by analyzing to what extent initially local operators become nonlocal. In analogy to the entangling power, wherein the action of operators on initially unentangled states is measured $[9,10]$, we may consider time evolution of initial product operators in the Heisenberg picture of quantum mechanics. Such an evolution is obtained as a special case of Eq. (46) if $U=V^{\dagger}$. This may be interpreted as the average entangling power on conjugation of product operators with the bipartite operator $V$. As $e_{p}\left(V^{\dagger}\right)=e_{p}(V)$, our results imply that

$$
\left\langle e_{p}\left[V^{\dagger}\left(u_{A} \otimes u_{B}\right) V\right]\right\rangle_{u_{A}, u_{B}}=e_{p}(V)\left[2-\frac{e_{p}(V)}{\overline{e_{p}}}\right] .
$$

This provides a way to quantify the scrambling power of bipartite unitary operators. It would be interesting to generalize such a scrambling power for a multipartite setup in analogy to the entangling power applied recently for several subsystems [60].

It might be surprising that simple relations (46) exist for $e_{p}$ and $g_{t}$, and this is due to the fact that they concern the average values. Similar relations hold for averaged operator entanglements $\left\langle E\left[U\left(u_{A} \otimes u_{B}\right) V\right]\right\rangle_{u_{A}, u_{B}}$ and $\left\langle E\left[U\left(u_{A} \otimes u_{B}\right) V S\right]\right\rangle_{u_{A}, u_{B}}$, but they mix among themselves in a less transparent way. Although the statements above concern averages over local unitaries, they provide some immediate insights. For instance, choosing $U$ and $V$ such that

$$
e_{p}(U)+e_{p}(V)-e_{p}(U) e_{p}(V) / \overline{e_{p}}>e_{p}(U V),
$$

we infer that there exist local unitaries that enhance the entangling power beyond a serial application of $V$ and $U$. Relations in Eq. (46) can be used to iterate by inserting independent local operators between nonlocal operators. For example, one becomes

$$
\begin{aligned}
\left\langle e_{p}[\right. & \left.\left.U\left(u_{A} \otimes u_{B}\right) V\left(u_{A}^{\prime} \otimes u_{B}^{\prime}\right) W\right]\right\rangle_{u_{A}, u_{B}, u_{A}^{\prime}, u_{B}^{\prime}} \\
= & e_{p}(U)+e_{p}(V)+e_{p}(W)-\left[e_{p}(U) e_{p}(V)\right. \\
& \left.+e_{p}(V) e_{p}(W)+e_{p}(W) e_{p}(U)\right] / \overline{e_{p}} \\
& +e_{p}(U) e_{p}(V) e_{p}(W) /\left(\overline{e_{p}}\right)^{2} .
\end{aligned}
$$

The above equation indicates a certain "decoupling" that is induced by local dynamics. It is necessary that the local operators at each product be independent, because correlations would prevent such an expression. However, previous work suggests [44] that they would provide a good approximation also if the matrices $u_{A}, u_{A}^{\prime}$ and $u_{B}, u_{B}^{\prime}$ are pairwise identical, provided they are Haar-typical random unitaries.

We now formally state the result concerning the thermalization of the entangling power and gate typicality averaged over random local dynamics in the generalized setting of unequal dimensions of the subsystems. The final formulas remain the same as those displayed in [44], indicating a certain universality in them. However, we present an alternate proof here based on irreducible representations of the unitary group. Due to the technical nature of the proof, we present the details separately in Appendix C.

Theorem V.1. Let $U$ and $V$ be bipartite unitary operators on $\mathcal{H}_{N}^{A} \otimes \mathcal{H}_{M}^{B}$, and let $u_{A}, u_{B}$ be sampled from the groups $U(N)$ and $U(M)$ of unitary matrices according to their Haar measures. Then the following relation 
holds:

$$
\begin{aligned}
& \left\langle e_{p}\left[U\left(u_{A} \otimes u_{B}\right) V\right]\right\rangle_{u_{A}, u_{B}} \\
& \quad=e_{p}(U)+e_{p}(V)-e_{p}(U) e_{p}(V) / \overline{e_{p}},
\end{aligned}
$$

where $\overline{e_{p}}=\left\langle e_{p}(W)\right\rangle_{W}$ denotes the mean entangling power averaged over random unitary matrices $W$ sampled according to the Haar measure on $U(N M)$.

Corollary V.1.1. Let $U^{(n)} \equiv U\left(u_{A_{n-1}} \otimes u_{B_{n-1}}\right) U \cdots\left(u_{A_{1}} \otimes\right.$ $\left.u_{B_{1}}\right) U$, where $u_{A_{j}} \in U(N)$ and $u_{B_{j}} \in U(M)$ are unitary matrices. Let $V=U^{(n-1)}$, so that $U^{(n)}=U\left(u_{A_{n-1}} \otimes u_{B_{n-1}}\right) V$. Then from the above theorem we obtain

$$
\begin{aligned}
\left\langle e_{p}\left(U^{(n)}\right)\right\rangle_{\mathrm{loc}} & =e_{p}(U)+\left[1-\frac{e_{p}(U)}{\overline{e_{p}}}\right]\left\langle e_{p}\left(U^{(n-1)}\right)\right\rangle_{\mathrm{loc}} \\
& =\overline{e_{p}}\left[1-\left(1-\frac{e_{p}(U)}{\overline{e_{p}}}\right)^{n}\right],
\end{aligned}
$$

where $\langle\cdot\rangle_{\text {loc }}$ denotes averaging over the set of local operators $u_{A_{n-1}}, u_{B_{n-1}} \cdots u_{A_{1}}, u_{B_{1}}$ generated independently according to the Haar measure.

Similarly, it follows that

$$
\left\langle g_{t}\left(U^{(n)}\right)\right\rangle_{\mathrm{loc}}=\bar{g}_{t}\left[1-\left(1-\frac{g_{t}(U)}{\overline{g_{t}}}\right)^{n}\right],
$$

where the average value $\bar{g}_{t}$ is provided in Eq. (47), and for $M=N$ it reduces to $1 / 2$.

A proof of this result is indicated in Appendix C.

Equations (51) and (53) constitute our main results concerning thermalization of properties of quantum gates iterated sequentially in discrete time steps. For any bipartite gate $U$ with arbitrarily small but positive entangling power, its repeated application with local unitaries sandwiched between consecutive time steps leads to a generic gate with entangling power and a gate typicality characteristic of the average over the ensemble of Haar random gates from $U(N M)$. The same is illustrated in Fig. 5 for qubits and qutrits in the $e_{p}-g_{t}$ plane. The evolution of $U^{n}$ and $\left\langle U^{(n)}\right\rangle_{\text {loc }}$ is shown for a particular (nongeneric) choice of the initial unitary $U$, which is selected from the vicinity of a local gate, as $e_{p}(U)$ and $g_{t}(U)$ are sufficiently small. While $U^{n}$ explores the set $K_{N}$ in a "billiard"-like dynamics [45], $\left\langle U^{(n)}\right\rangle_{\text {loc }}$ converges exponentially to the CUE average.

Our results involve averaging over different local operators at each time step and may be considered a foil for quantities such as $e_{p}\left\{\left[\left(u_{A} \otimes u_{B}\right) U\right]^{n}\right\}$ if $u_{A, B}$ are sufficiently random and have no special relationship with $U$. Thus while the above results may be applicable for nonautonomous Floquet systems, they are also of relevance to autonomous ones. In the case of a many-body spin chain, the effect of thermalization of the average entangling power to equilibrium has recently been reported [14] for the symmetric case of $N=M$. The generalization presented here allows us to extend such studies of thermalization to the important case of different numbers of spins in each subsystem.

\section{Example: Random diagonal nonlocal operators}

In [44] the entangling powers of $U^{n}$ and $U^{(n)}$ were evaluated for a few gates $U$ for the symmetric case $M=N$. Here we augment these results significantly by numerically
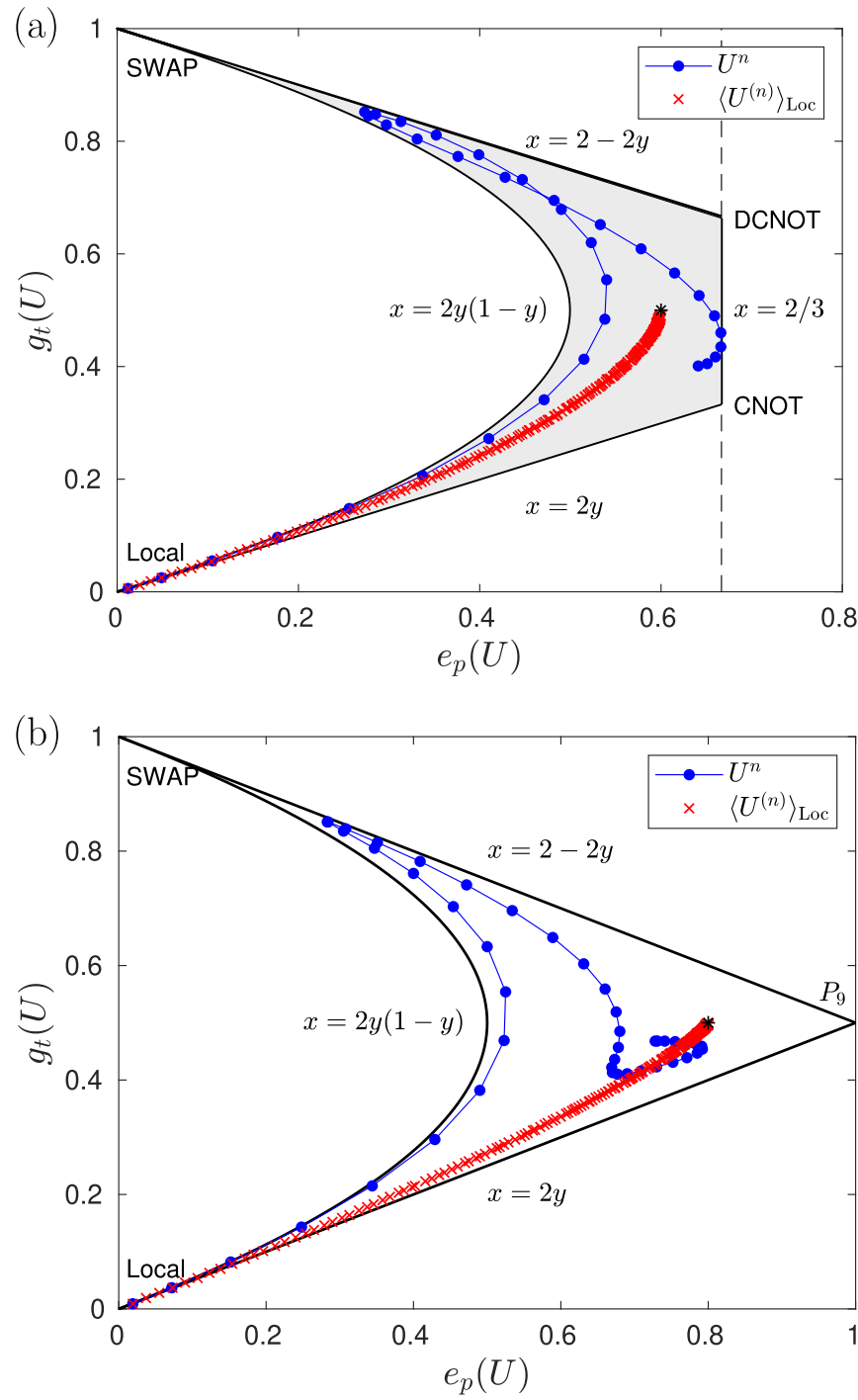

FIG. 5. Exemplary dynamics in the set $K_{N}$ starting in the vicinity of a local gate for (a) $N=2$ and (b) $N=3$. The average value $\left\langle U^{(n)}\right\rangle_{\text {loc }}$, represented by red crosses $(\times)$, saturates to the CUE average (*), while $U^{n}$ follows a billiard-type dynamics [45]. The blue line joining the points in $U^{n}$ is added to guide the eye. At each time step, the average is taken over $10^{4}$ local random unitaries.

showing that for $N \neq M$, the thermalization of the entangling power to its average value $\overline{e_{p}}$ holds also in the case of a very small interaction between both subsystems. In particular, we analyze below the simplest interesting case of a qubit-qutrit system.

Consider a diagonal unitary matrix on $\mathcal{H}_{N}^{A} \otimes \mathcal{H}_{M}^{B}$ with entries

$$
\left(U_{\epsilon}\right)_{m \alpha ; n \beta}=e^{2 \pi i \epsilon \xi_{m \alpha}} \delta_{m n} \delta_{\alpha \beta},
$$

where $\epsilon \in[0,1]$, and $\xi$ is chosen randomly and uniformly from $[-1 / 2,1 / 2)$. Such diagonal unitaries are used to model interactions in several deterministic Floquet operators $[24,74,75]$. While $\epsilon=0$ is evidently the case of zero interaction, $\epsilon=1$ represents the maximal interaction. As $\xi$ is a random variable, $\left(U_{\epsilon}\right)_{m \alpha ; n \beta}$ defines an ensemble of entangling gates. Their entangling power was studied in [76] for the 


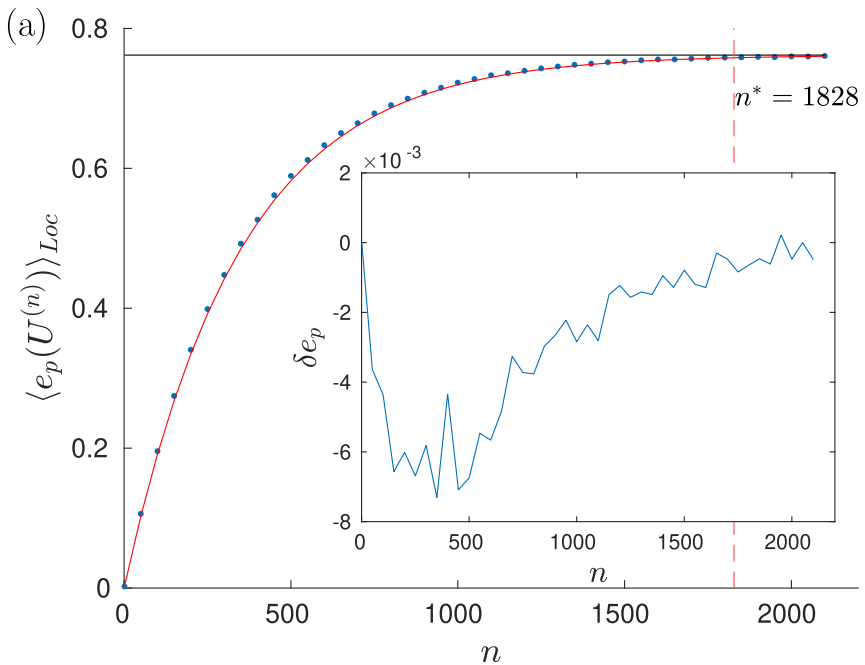

(b) 0.8

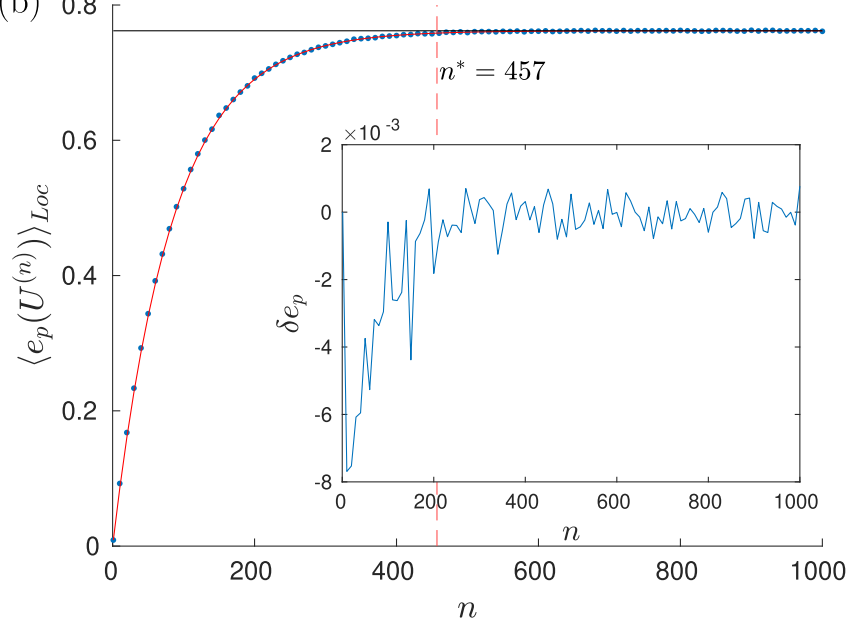

FIG. 6. Saturation of entangling power $e_{p}$ for subsystems of size $N=2$ and $M=3$ as a function of discrete time $n$ for two values of the interaction parameter $\epsilon, \epsilon=0.025$ and 0.05 in (a) and (b), respectively. At each time step, the average is taken over $10^{4}$ local random unitaries. The solid curve represents Eq. (52), while the horizontal line denotes the Haar average $\overline{e_{p}}$ given by Eq. (47). Saturation timescales as $1 / \epsilon^{2}$; see (58). The inset shows deviations from the theoretical values.

case $\epsilon=1$, while for general $\epsilon$ it has been used in studies of spectral transitions and entanglement [24,74,75].

For a fixed realization of the global diagonal $U_{\epsilon}$, even if $\epsilon$ is very small, $\left\langle e_{p}\left(U_{\epsilon}^{(n)}\right)\right\rangle_{\text {loc }}$ reaches the Haar average $\overline{e_{p}}$ due to interlacing of random local unitaries as illustrated in Fig. 6 in the case $M N=6$. For $e_{p}\left(U_{\epsilon}\right) \ll 1$, Eq. (52) implies that

$$
\left\langle e_{p}\left(U_{\epsilon}^{(n)}\right)\right\rangle_{\mathrm{loc}} \approx \overline{e_{p}}\left[1-\exp \left(-n \frac{e_{p}\left(U_{\epsilon}\right)}{\overline{e_{p}}}\right)\right] .
$$

The saturation value reached is equal to the global average $\overline{e_{p}}$, which according to Eq. (47) reads $16 / 21 \approx 0.762$ for $N=2$ and $M=3$. The smaller the interaction parameter, the longer it takes to thermalize and reach the asymptotic value. Deviations from the theoretical curve shown in the insets of Fig. 6 are of the order of $1 / \sqrt{n_{\text {loc }}}$, where $n_{\text {loc }}$ denotes the number of realizations of local gates over which the averaging is done.
Hence the number of locals $n^{*}$ required to push $e_{p}\left(U_{\epsilon}^{(n)}\right)$ to the Haar average depends on $\epsilon$ as $n^{*} \sim \overline{e_{p}} / e_{p}\left(U_{\epsilon}\right)$.

The time of thermalization can be estimated for the case of local evolution given by the tensor product of diagonal random gates. For a diagonal unitary $U_{\epsilon}$ of size $N M \times N M$, the reshuffled matrix is of size $N^{2} \times M^{2}$ with $N(N-1)$ rows and $M(M-1)$ columns equal to zero. To compute $\operatorname{tr}\left(U^{R}\left(U^{R}\right)^{\dagger}\right)^{2}$ in Eq. (45), it is thus sufficient to consider $\operatorname{tr}\left(A A^{\dagger}\right)^{2}$, where $A$ is obtained by reshaping the diagonal of $U_{\epsilon} ; A_{j k}=e^{2 \pi i \epsilon \xi_{\alpha}}$, $\alpha=(j-1) M+k, j=1, \ldots, N, k=1, \ldots, M$. Here $A A^{\dagger}$ is a Hermitian matrix of size $N$, and for $\epsilon$ small, $\left(A A^{\dagger}\right)_{j j}=M$ and off-diagonal entries $\left(A A^{\dagger}\right)_{j k} \approx M(1 \pm i \epsilon), j<k$. Thus,

$$
\operatorname{tr}\left(U_{\epsilon}^{R}\left(U_{\epsilon}^{R}\right)^{\dagger}\right)^{2}=\operatorname{tr}\left(A A^{\dagger}\right)^{2} \approx N^{2} M^{2}-N(N-1) M^{2} \epsilon^{2} .
$$

The partial transpose of a diagonal unitary remains unchanged, hence

$$
\operatorname{tr}\left(U_{\epsilon}^{T_{A}}\left(U_{\epsilon}^{T_{A}}\right)^{\dagger}\right)^{2}=N M
$$

Inserting Eqs. (56) and (57) into Eq. (45) gives $e_{p}\left(U_{\epsilon}\right) \approx$ $N \epsilon^{2} /(N+1)$, and therefore

$$
n^{*} \approx \frac{(N+1)\left(M^{2}-1\right)}{M(N M+1)} \frac{1}{\epsilon^{2}},
$$

which for $N=2, M=3$ gives $n^{*} \approx 8 \epsilon^{-2} / 7$. For $\epsilon=0.025$ and 0.05 , the numerical values read $n^{*} \approx 1828$ and $n^{*} \approx 457$, respectively, as shown in Fig. 6.

For large dimensions $M$ and $N$, one may average additionally over the diagonal ensemble of the entangling gates themselves. It is possible to approximate such an ensemble-averaged $\operatorname{tr}\left(A A^{\dagger}\right)^{2}$ by $N^{2} M^{2} \operatorname{sinc}^{4}(\pi \epsilon) \approx(1-$ $\left.2 \pi^{2} \epsilon^{2} / 3\right) N^{2} M^{2}$, where $\operatorname{sinc}(x)=\sin x / x$. Hence $e_{p}\left(U_{\epsilon}\right) \approx$ $2 \pi^{2} \epsilon^{2} / 3$ and $n^{*} \sim 3 \overline{e_{p}} /\left(2 \pi^{2} \epsilon^{2}\right)$, so that the saturation time scales as $1 / \epsilon^{2}$.

Time evolution of quantum entanglement for initially separable states has been the subject of many studies [16-20,30], often in the context of weakly interacting highly chaotic systems. A recent study [32] combining a recursive application of perturbation theory and the theory of random matrices indicates an exponential saturation of entanglement measures and is consistent with our findings. The approach advocated here is not perturbative, and it is based on averaging the entangling power over independent local operators at each time step. The rate at which the average $\left\langle e_{p}\left(U^{(n)}\right)\right\rangle$ approaches the global RMT value $\bar{e}_{p}$ depends only on the entangling power of the nonlocal single-step operator $U$ and is hence fully interactiondriven.

Note that the techniques applied in this work are not sensitive to the degree of chaos in the classical model consisting of two uncoupled systems. Thus analyzing the time evolution of averaged entangling power, we are not in a position to investigate the role of the Lyapunov exponent of the corresponding classical system, which was found to be essential [30] for the rate of growth of the average entanglement of quantum states initially localized in phase space. The fact that the entangling power averages over all initial product states equally implies that any special properties that arise for coherent initial states are washed out. However, further work is needed to clarify the connections and differences between both approaches. 


\section{B. Thermalization of the spectra of reshuffled and partially transposed unitaries}

We have analyzed above how the local unitary invariants of entangling power and operator entanglement, and equivalently the entropies of the density matrices in (21), thermalize in time to their asymptotic values. However, this only reflects a more detailed approach to equilibrium of the spectra of related operators. In particular, it is illuminating to analyze complex eigenvalues of nonunitary reshuffled and partially transposed matrices, $U^{(n) R}$ and $U^{(n) T_{A}}$, which allow us to infer to what extent the analyzed gate approaches properties characteristic of generic unitary matrices.

A large non-Hermitian random matrix $G$ from the Ginibre ensemble, containing independent complex random Gaussian entries, displays a spectrum covering uniformly the unit disk, according to the universal circular law of Girko [47,77]. If a random unitary matrix $U$ is large enough, the unitarity constraints become so weak that after reshuffling the matrix $U^{R}$ shows statistical properties close to these of the Ginibre ensemble $[45,49]$ — see also the recent rigorous results in Ref. [78]. Thus the corresponding positive matrix, $U^{R}\left(U^{R}\right)^{\dagger}$, displays spectra in agreement with the Marčenko-Pastur law [48], $P_{\mathrm{MP}}(x)=(2 \pi)^{-1} \sqrt{(4-x) / x}$, derived to describe the spectral density of random Wishart matrices $G G^{\dagger}$.

Let $x_{i}$ denote eigenvalues of the density matrices $\rho_{R}\left(U^{(n)}\right)$ or $\rho_{T}\left(U^{(n)}\right)$ rescaled by the dimension $N^{2}$, which are equal to scaled squared singular values of $\left(U^{(n)}\right)^{R}$ and $\left(U^{(n)}\right)^{T_{A}}$, respectively. The thermalization of properties of the gate $U^{(n)}$ with time $n$ will be reflected in the distribution $P(x)$, which for a large dimension $N$ converges to the distribution $P_{\mathrm{MP}}(x)$. We will introduce local averaged purities of both auxiliary density matrices,

$$
X_{n}=\left\langle\operatorname{tr}\left[\rho_{R}^{2}\left(U^{(n)}\right]\right\rangle, \quad Y_{n}=\left\langle\operatorname{tr}\left[\rho_{T}^{2}\left(U^{(n)}\right)\right]\right\rangle .\right.
$$

Then the Marčenko-Pastur law implies that $X_{n}$ and $Y_{n}$ are of the order of $2 / N^{2}$. A recursion relation for these quantities starting from $\left(X_{1}, Y_{1}\right)$ was derived in [44] for the symmetric case, $M=N$. We will now demonstrate thermalization in the spectra of density operators $\rho_{R}$ and $\rho_{T}$ for the model of the diagonal unitary ensemble and controlled unitaries.

\section{Spectral properties for random diagonal nonlocal operators}

Consider the special case of the model with nonlocal matrix $U$ being diagonal with random phases, as in Eq. (54). To focus on the effect of time evolution itself, we set the interaction strength to the maximal value, $\epsilon=1$, choose $N=M$, and denote the diagonal nonlocal matrix by $U=U_{d}$. Average purities of the density matrices defined in Eq. (59) for $n=1$ read

$$
\overline{X_{1}}=\frac{2 N-1}{N^{2}} \text { and } \overline{Y_{1}}=\frac{1}{N^{2}} .
$$

In this case, there are no local operators and the averaging indicates only the average with respect to the random phases of the nonlocal operators $U_{d}$. The first one is easy to derive from the reshuffled operator (see [76]), and since the partial transpose of a diagonal unitary matrix remains diagonal, $\rho_{T_{A}}\left(U_{d}\right)=I_{N^{2}} / N^{2}$, hence $Y_{1}=1 / N^{2}$. Thus typical diagonal unitaries, even for $\epsilon=1$, are far from being thermalized, although their entangling power $e_{p}\left(U_{d}\right)=(N-1) /(N+1)$ is large. This follows from Eq. (15); see also [76], in which a different normalization of the entangling power is used.

For $n=2$ we consider an interlacing dynamics determined by random local unitary operators acting between two nonlocal operators, $U^{(2)}=U_{d}\left(u_{1} \otimes u_{2}\right) U_{d}$, and we obtain

$$
\overline{X_{2}}=\frac{6}{N^{2}+1} \text { and } \overline{Y_{2}}=\frac{2\left(N^{4}+N^{2}+1\right)}{N^{4}(N+1)^{2}} .
$$

Since $\overline{Y_{2}} \sim 2 / N^{2}$, this quantity related to the partial transpose of $U_{d}$ is close to its asymptotic value already after two applications of typical nonlocal diagonal operators. On the other hand, the dual quantity $\overline{X_{2}}$ behaves as $6 / N^{2}$, which indicates significant deviations from typicality.

These effects are visible in Fig. 7 in multiple ways. The eigenvalues of $\left(U_{d}^{(2)}\right)^{R}$ are not distributed uniformly inside the unit disk, which is the case for the spectrum of $\left(U_{d}^{(2)}\right)^{T_{A}}$. For the former operator there are several small eigenvalues, which reflects the fact at $n=1$, the matrix $U_{d}^{R}$ is of rank $N$, rather than $N^{2}$. Even in the case of the partial transpose, there are visible deviations from the linear structure of the radial distribution that are not observed for $n \geqslant 3$. Although $\overline{X_{3}} \sim 2 / N^{2}$, there exist deviations in the radial distribution, which thus serve as a sensitive indicator of thermalization. At $n=4$, the properties of the partial transpose and the reshuffled matrix are close to the matrices from the Ginibre ensemble of dimension $N^{2}$, and the singular values follow the Marčenko-Pastur law to a good approximation.

\section{Spectral properties for controlled unitary operators}

While the diagonal nonlocal operator led to fast thermalization, for some other models this process occurs considerably slower. Consider a controlled unitary operator acting on a symmetric product space,

$$
U=P_{A_{1}} \otimes \mathbb{1}_{B}+P_{A_{2}} \otimes u_{B},
$$

where $P_{A_{i}}$ are orthogonal projectors such that $P_{A_{1}}+P_{A_{2}}=\mathbb{1}_{N}$, $P_{A_{i}} P_{A_{j}}=\delta_{i j} P_{A_{i}}$, and $u_{B} \in U(N)$. It is known [79] that any two-qubit unitary gate of Schmidt rank 2 forms a controlledunitary of this kind, and it can be implemented with a maximally entangled state of two qubits and local operations and classical communication (LOCC). Thus this example may be considered the simplest entangling unitary. The reshuffled operator reads

$$
U^{R}=\left|P_{A_{1}}^{R}\right\rangle\left\langle\mathbb{1}^{R}|+| P_{A_{2}}^{R}\right\rangle\left\langle u_{B}^{* R}\right|,
$$

where $|M\rangle$ reshapes or vectorizes the operator with elements $M_{i j}=\langle i|M| j\rangle$ into a column vector with entries $M_{i j}$. Noting that $\left\langle M_{1} \mid M_{2}\right\rangle=\operatorname{tr}\left(M_{1}^{\dagger} M_{2}\right)$, we get

$$
\begin{aligned}
\rho_{R}= & \frac{1}{N^{2}} U^{R} U^{R \dagger}=\frac{1}{N^{2}}\left[N\left(\left|P_{A_{1}}^{R}\right\rangle\left\langle P_{A_{1}}^{R}|+| P_{A_{2}}^{R}\right\rangle\left\langle P_{A_{2}}^{R}\right|\right)\right. \\
& \left.+\operatorname{tr}\left(u_{B}\right)^{*}\left|P_{A_{1}}^{R}\right\rangle\left\langle P_{A_{2}}^{R}\left|+\operatorname{tr}\left(u_{B}\right)\right| P_{A_{2}}^{R}\right\rangle\left\langle P_{A_{1}}^{R}\right|\right],
\end{aligned}
$$

which is only a rank- 2 operator. In contrast, as $\left(u_{A} \otimes u_{B}\right)^{T_{A}}=$ $u_{A}^{T} \otimes u_{B}$ and as transposes of projectors remain projectors, $U^{T_{A}}$ is also unitary and hence $\rho_{T}=\mathbb{1}_{N^{2}} / N^{2}$, a maximally 
(a)
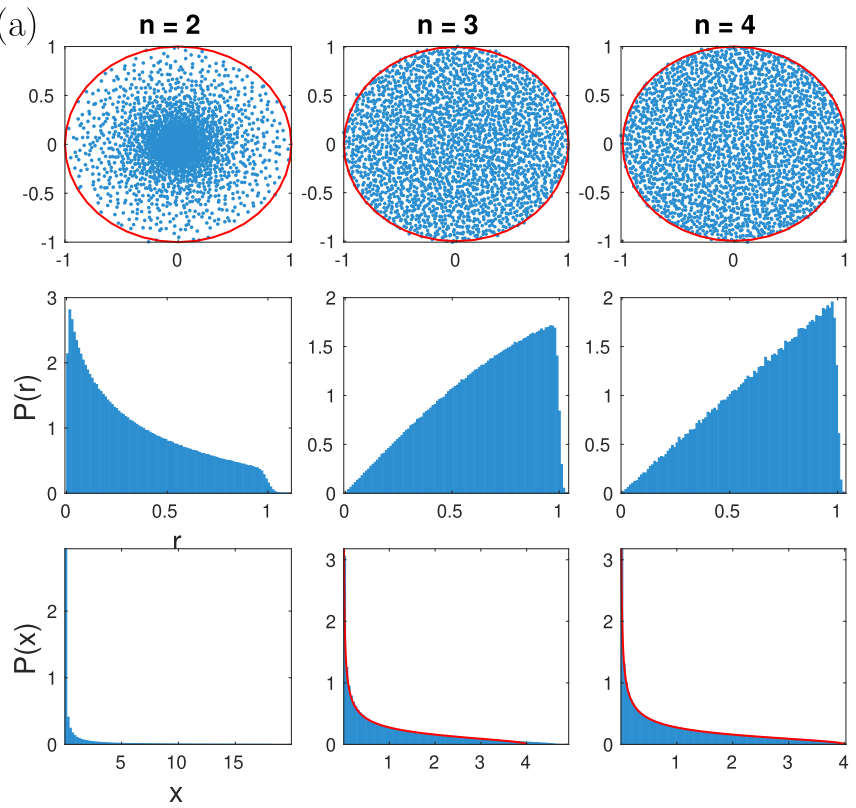

(b)
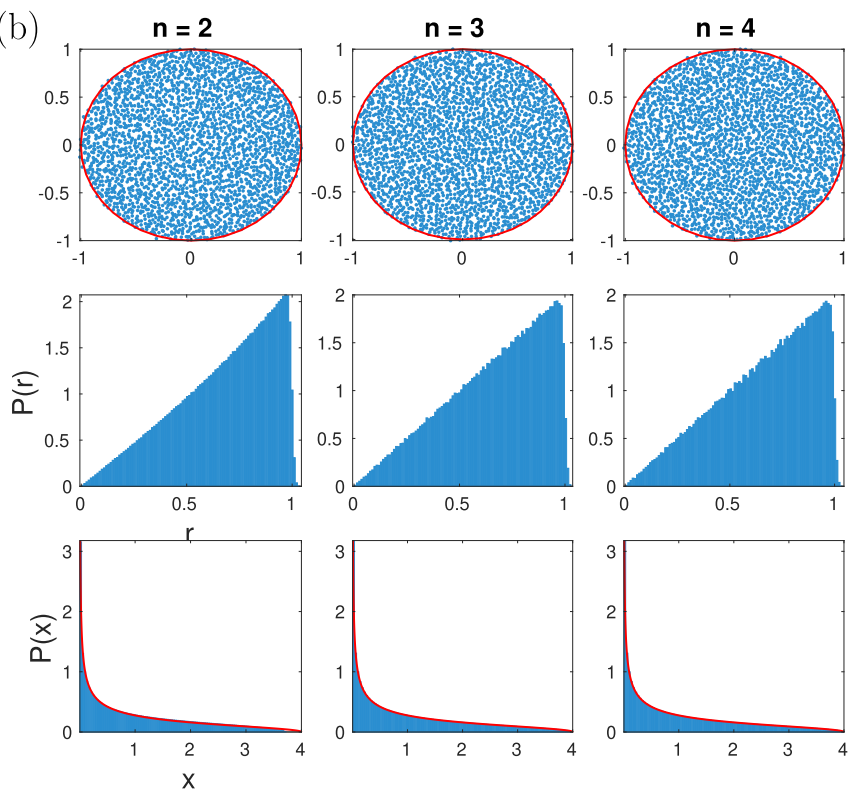

FIG. 7. Spectral thermalization of $U^{(n)}$ for a random diagonal nonlocal unitary matrix $U_{d}$ of dimension $N^{2}$ with $N=50$. Distributions corresponding to (a) of reshuffled matrix $\left(U^{(n)}\right)^{R}$ and (b) the partially transposed matrix $\left(U^{(n)}\right)^{T_{A}}$ are shown for times $n=2,3$, and 4. Top rows show the complex eigenvalues $z$, while middle rows show their radial density, $P(r)$, with $r=|z|$. The bottom rows show the distribution of scaled squared singular values of $\left(U^{(n)}\right)^{R}$ and $\left(U^{(n)}\right)^{T_{A}}$, respectively, which are compared with the Marčenko-Pastur distribution (solid curve).

mixed state. These observations immediately imply that

$$
X_{1}=\frac{1}{2}+\frac{1}{2 N^{2}}\left|\operatorname{tr}\left(u_{B}\right)\right|^{2} \quad \text { and } \quad Y_{1}=\frac{1}{N^{2}} .
$$

Taking Haar random unitary matrices $u_{B}$ of size $N$, one defines an ensemble of controlled-unitary gates of order $N^{2}$, for which we evaluate the average purities of the associated density matrices $\rho_{R}\left(U^{(n)}\right)$ and $\rho_{T}\left(U^{(n)}\right)$. The form factor averaged over CUE matrices of size $N$ reads $\overline{\left|\operatorname{tr}\left(u_{B}^{n}\right)\right|^{2}}=n$ if $n \leqslant N$ and $N$ for $n \geqslant N$-see [80]. Denoting this additional averaging by an overbar, one obtains $\overline{X_{1}} \sim 1 / 2$ and $\overline{Y_{1}}=1 / N^{2}$. Using the recursion relation from [44] and the CUE form factors quoted above for $n=2$, we arrive at

$$
\begin{aligned}
& \overline{X_{2}}=\frac{N^{6}+2 N^{4}-6 N^{2}+4}{4 N^{2}\left(N^{2}-1\right)^{2}}, \\
& \overline{Y_{2}}=\frac{5 N^{4}-10 N^{2}+6}{4 N^{2}\left(N^{2}-1\right)^{2}} .
\end{aligned}
$$

The other details of the unitary gate $u^{B}$ are relevant to higher orders. It is also clear that the sequence $Y_{n}$ approaches the typical behavior earlier than the $X_{n}$. For instance, at $n=2$ we have $\overline{X_{2}} \sim 1 / 4$, while $\overline{Y_{2}} \sim \frac{5}{4 N^{2}}$. In general, $\overline{X_{n}} \sim \frac{1}{2^{n}}$, indicating that it takes a time $n^{*} \sim 4 \log _{2} N$ for the operators to thermalize, such that the average operator entanglement is comparable to the average over $U\left(N^{2}\right)$. Numerical data obtained for a typical controlled unitary gate presented in Fig. 8 show that in this case the thermalization time is longer in comparison to random diagonal gates. Even at $n=12$ one can see substantial deviations from the Girko circular law for the eigenvalues of the reshuffled matrix, while at $n=14$ the data become well described by the universal Marčenko-Pastur distribution. Spectral properties of the partially transposed matrix also reach typical behavior around $n \sim 10$. These timescales are consistent with the $\log _{2} N$ scale, and the thermalization of the controlled-unitary gates occurs more slowly, but surely.

\section{SUMMARY AND OUTLOOK}

In this work, we have investigated the nonlocal properties of bipartite quantum gates acting on an $N \times M$ system. Representing them in the plane spanned by entangling power $e_{p}$ and gate typicality $g_{t}$, we have analyzed the boundary of the allowed set $K_{2}$, which in turn enabled us to identify gates that correspond to critical points of the boundary and are distinguished by some particular properties. Making use of the Cartan decomposition and the canonical form of a two-qubit gate $[62,63]$ we have described the boundaries analytically, as they correspond to the edges and diagonals of the Weyl chamber.

As the Cartan decomposition is not effective for unitary matrices of order 9, in the case of two-qutrit gates such an approach does not work, hence only some parts of the boundary of the set $K_{3}$ are known exactly. For instance, the structure of $K_{3}$ is still unknown in the vicinity of the rightmost point representing optimal gates, for which entangling power admits its maximal value, $e_{p}=1$, and it corresponds to maximally entangled states of a four-qutrit system. It is worth emphasizing that while such a gate does not exist for $N=2$ [46], the case of $N=6$ still remains open [81].

A key issue addressed in this paper concerns the nonlocal properties of a bipartite unitary gate applied sequentially. Although local unitary operations performed after a single usage of a nonlocal gate cannot change its entangling power, they do play a crucial role if the gate analyzed is performed several times. Our result shows that an arbitrarily small, but positive, entangling power of a nonlocal gate $U_{A B}$ is sufficient to assure that the gate $U_{A B}^{\prime}=U_{A B} V_{\text {loc }}$ applied $n$ times will 
(a)
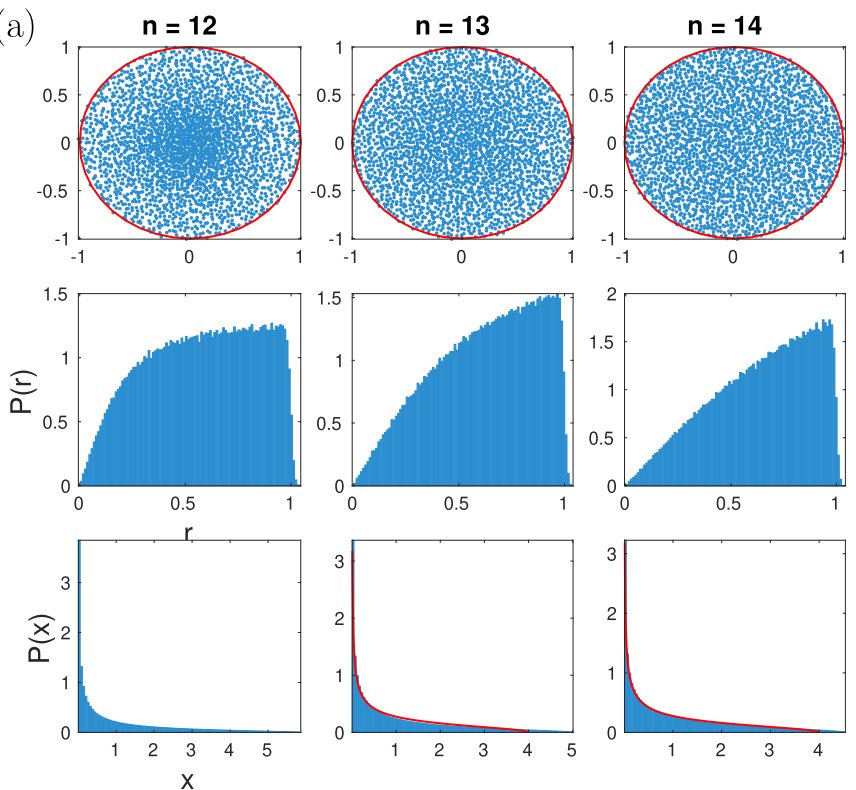

(b)
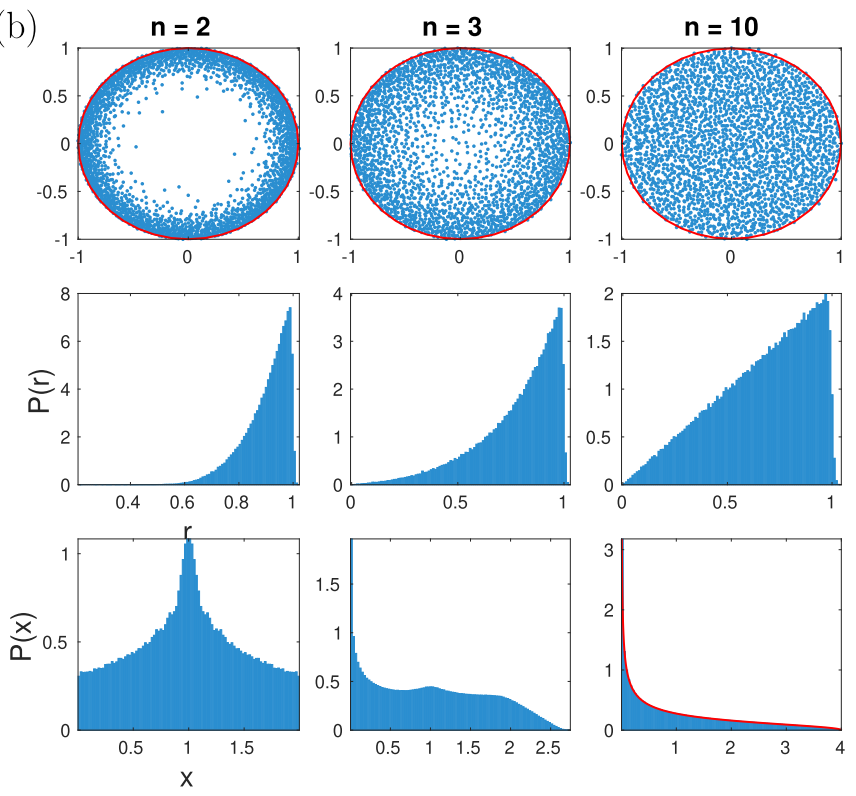

FIG. 8. As in Fig. 7 for $U$ selected as a controlled unitary gate. Third column plotted for $n=14$ and 10 illustrates the longer time required in this case to reach typicality.

reach the entangling power typical for random unitary matrices exponentially fast. Here $V_{\mathrm{loc}}=u_{A} \otimes u_{B}$ denotes a random local unitary, which is drawn independently at each time step. This statement illustrates the thermalization of nonlocal properties of bipartite gates with the interaction time and sheds more light on the properties of quantized chaotic dynamics, in which nonlocal kicks coupling both subsystems are interlaced by chaotic local evolution [82].

While the entangling power of a bipartite gate $U_{A B}$ determines the entangling power of time evolutions augmented with local operators, it is interesting to note that it can possibly determine the complexity of the corresponding many-body systems built out of them in various architectures. As a concrete example, a product of $\otimes^{L} U_{A B}$ on a one-dimensional lattice of $2 L$ sites and its translation by one site was studied recently in terms of its correlation functions [54]. It is not hard to infer from their results that the case when $U_{A B}$ has maximal entangling power allowed by the dimensions corresponds to the case of a maximally chaotic many-body system. It is interesting to observe that qubits do not satisfy this condition, while this is the case for qutrits [69]. Thus we believe that our study is also relevant for a large body of recent work around understanding quantum chaos for many-body systems.

\section{ACKNOWLEDGMENTS}

We would like to thank Wojciech Bruzda and Dardo Goyeneche for several fruitful discussions, and Suhail Ahmad Rather for crucial help with the qutrit phase-space. K.Ż. acknowledges financial support by Narodowe Centrum Nauki under Grant No. DEC-2015/18/A/ST2/00274. A.L. and P.M. acknowledge financial support by the Department of Science and Technology, Govt. of India, under Grant No. DST/ICPS/QuST/Theme-3/2019/Q69.

\section{APPENDIX A: BIPARTITE UNITARY GATES AND FOUR-PARTY ENTANGLED PURE STATES}

The bijection between states on $\mathcal{H}^{(1)} \otimes \mathcal{H}^{(2)}$ and operators on $\mathcal{H}^{(1)} \cong \mathcal{H}^{(2)}$ is known in the physics literature as Choi-Jamiołkowski isomorphism, which relates the set of pure states of a bipartite system and the set of operations acting on a simple system [83]. Any normalized bipartite pure state $|\psi\rangle=\sum_{i j=1}^{N} x_{i j}|i j\rangle$ can be written as $(X \otimes \mathbb{1})\left|\phi^{+}\right\rangle$, where $\left|\phi^{+}\right\rangle=\sum_{j=1}^{N}|j j\rangle / \sqrt{N}$ is the maximally entangled state and $\langle i|X| j\rangle=\sqrt{N} x_{i j}$. Note that a state $|\psi\rangle$ is maximally entangled if and only if the matrix $X$ is unitary, as then its partial trace is maximally mixed, $\rho_{A}=\operatorname{tr}_{B}|\psi\rangle\langle\psi|=X X^{\dagger} / N=\mathbb{1} / N$. It is often convenient to make use of this relation between the set $U(N) / U(1)$ of unitary quantum gates of order $N$ and the set of maximally entangled states in an $N \times N$ system [83].

The same relation can also be used in a more general setup if the system $\mathcal{H}^{(1)}$ is a composite and describes two subsystems of sizes $N$ and $M \geqslant N$, denoted $A$ and $B$, respectively. The system $\mathcal{H}^{(2)}$ of the same size $N M$ is also a composite and contains two subsystems $C$ and $D$ of dimensions $N$ and $M$, respectively. The matrix $X / \sqrt{N M}$ with elements $x_{i \alpha, k \beta}$ describes now a four-party pure state $\left|\psi_{A B C D}\right\rangle=\sum_{i j}^{N} \sum_{\alpha \beta}^{M} x_{i \alpha, k \beta}|i \alpha k \beta\rangle$, and it can be considered as a four-index tensor or an $N M \times$ $N M$ matrix with composite indices.

Any bipartite matrix $X$ acting on subsystems $A B$ thus defines a four-partite pure state,

$$
\left|\psi_{A B C D}\right\rangle=\left(X_{A B} \otimes \mathbb{1}_{C D}\right)\left|\phi_{A C}^{+}\right\rangle \otimes\left|\phi_{B D}^{+}\right\rangle .
$$

Note that the above formula does not factorize, as the symbol $\otimes$ denotes tensor products acting with respect to different partitions. If the bipartite matrix acting on the subsystems $A B$ is unitary, $X=U$, then the corresponding four-party state $\left|\psi_{A B C D}\right\rangle$ is maximally entangled with respect to the partition $A B \mid C D$, so all the components of the corresponding Schmidt vector of length $N M$, eigenvalues of $\rho_{A B}=\operatorname{tr}_{C D}\left|\psi_{A B C D}\right\rangle\left\langle\psi_{A B C D}\right|=U U^{\dagger} / N M$, are equal to $1 / N M$. The unitarity condition, $U U^{\dagger}=\mathbb{1}$, implies then the maximal 
entanglement of the state $\left|\psi_{A B C D}\right\rangle$ with respect to the splitting $A B \mid C D$.

On the other hand, one can investigate whether this state is entangled with respect to two other possible partitions, $A C \mid B D$ and $A D \mid B C$. To this end, one studies the partially reduced states $\rho_{A C}=\operatorname{tr}_{B D}\left|\psi_{A B C D}\right\rangle\left\langle\psi_{A B C D}\right|$ with spectrum $\lambda_{i}$ with $1 \leqslant i \leqslant N^{2}$, and $\rho_{A D}=\operatorname{tr}_{B C}\left|\psi_{A B C D}\right\rangle\left\langle\psi_{A B C D}\right|$ with spectrum $\mu_{j}$ with $1 \leqslant j \leqslant N M$.

For any four-index matrix $X_{i j, \alpha \beta}$ of size $N M$ it will be convenient to use the following operations on its entries [83]: the partial transpose, $X^{T_{A}}$, where $X_{i \beta, j \alpha}^{T_{A}}=X_{i \alpha, j \beta}$, is also an $N M \times N M$ matrix, and the reshuffling, $X^{R}$, where $X_{i j, \alpha \beta}^{R}=$ $X_{i \alpha, j \beta}$ is an $\left(N^{2} \times M^{2}\right)$-dimensional array. The first one, $T_{A}$, represents transposition on the first subsystem only and preserves hermiticity of $X$. The reshuffling $R$, corresponding to reshaping each block of a matrix into a vector, does not preserve unitarity nor hermiticity.

It is easy to check [12] that the vector $\lambda$, equal to the spectrum of the positive matrix

$$
\rho_{R}(U) \equiv \rho_{A C}=U^{R}\left(U^{R}\right)^{\dagger} /(N M),
$$

coincides with the vector defining the operator Schmidt decomposition of the scaled matrix $U$. Correspondingly, the vector $\mu$, forming the spectrum of

$$
\rho_{T_{A}}(U) \equiv \rho_{A D}=U^{T_{A}}\left(U^{T_{A}}\right)^{\dagger} /(N M),
$$

appears in the Schmidt decomposition of the operator $U$ composed with the SWAP $S$ for the symmetric case.

The reduced state of the subsystems $A C$ is maximally mixed if $\rho_{A C}=\mathbb{1}_{N^{2}} / N^{2}$ and corresponds to the maximum entanglement in the $A C \mid B D$ split. This in turn happens when the rearrangement $U^{R}$ satisfies $U^{R}\left(U^{R}\right)^{\dagger}=\mathbb{1}_{N^{2}} /(M N)$, where $\mathbb{1}_{N^{2}}$ is the identity matrix of dimension $N^{2}$. In other words, for the symmetric case $N=M$, if $U^{R}$ is also unitary, then $\rho_{R}(U)$ is maximally mixed and the subsystem $A C$ is maximally mixed with $B D$. Hence the linear entanglement entropy $1-\operatorname{tr}_{A C} \rho_{A C}^{2}$, based on the reshuffling of $U$, can serve as a measure of the entanglement in the four-party state in Eq. (A1) with respect to the partition $A C \mid B D$.

The state $\left|\psi_{A B C D}\right\rangle$ is maximally entangled with respect to the third splitting $A D \mid B C$ if the Schmidt vector is flat, $\mu_{j}=$ $1 / M N$ for $j=1, \ldots, N M$, so that the matrix $X^{T_{A}}$ is unitary. Entanglement for this partition can thus be characterized by the twin quantity $1-\operatorname{tr}_{A C} \rho_{A D}^{2}$.

Analyzing a bipartite unitary gate $U$, described by a fourindex matrix $U_{a, b}=X_{i \alpha, j \beta}$, it is convenient to introduce the notion of multiunitarity [58]. For the symmetric case $(N=$ $M$ ), a matrix $X$ of size $N^{2}$, written in the four-index notation, is called 2-unitary, if the following three conditions are satisfied:

(i) $X \in U\left(N^{2}\right) \Leftrightarrow \sum_{i j} X_{i j, \alpha \beta} \bar{X}_{i j, \alpha^{\prime} \beta^{\prime}}=\delta_{\alpha, \alpha^{\prime}} \delta_{\beta, \beta^{\prime}}$,

(ii) $X^{R} \in U\left(N^{2}\right) \Leftrightarrow \sum_{i, \alpha} X_{i j, \alpha \beta} \bar{X}_{i j^{\prime}, \alpha \beta^{\prime}}=\delta_{j, j^{\prime}} \delta_{\beta, \beta^{\prime}}$,

(iii) $X^{T_{A}} \in U\left(N^{2}\right) \Leftrightarrow \sum_{i, \beta} X_{i j, \alpha \beta} \bar{X}_{i j^{\prime}, \alpha^{\prime} \beta}=\delta_{j, j^{\prime}} \delta_{\alpha, \alpha^{\prime}}$,

so apart from $U$, two other matrices with interchanged entries, $U^{T_{A}}$ and $U^{R}$, are also unitary. The corresponding four-index tensor $X_{i \alpha, j \beta}$ of size $N$ is called perfect if for any choice of two indices out of four the matrix of size $N^{2}$ obtained by restructuring the four-index tensor into a matrix is unitary [84].
By construction, any 2-unitary matrix $U$ of order $N^{2}$ provides an example of a matrix that maximizes the entangling power, $e_{p}(U)=1$, as both linear entanglement entropies $E(U)$ and $E(U S)$ are maximal. Thus the corresponding four-party state (A1) is maximally entangled with respect to all three possible partitions. Such states are called two-uniform [85] or absolutely maximally entangled (AME) [59].

Interestingly, such states do not exist in a four-qubit system [46], as the total size of the Hilbert space is too small to find a state satisfying all necessary constraints. This is equivalent to the known fact $[9,69]$ that there is no unitary matrix of size $N^{2}=4$ for which the maximal value $e_{p}=1$ of the entangling power is achieved, which is consistent with the structure of the set $K_{2}$ plotted in Fig. 1. In the complementary notation, there are no 2-unitary matrices of order four [58]. On the other hand, AME states exists for larger systems consisting of four qutrits, which is equivalent to the statement that there exists a 2-unitary matrix of size $N^{2}=9$, which maximizes the entangling power $e_{p}$ [69]. For any $N=3,4,5$ and $N \geqslant 7$, there exist permutations matrices of size $N^{2}$ that are 2-unitary, and hence maximize the entangling power [69] and also correspond to AME states of four systems with $N$ levels each. For $N=6$, the nonexistence of any 2-unitary permutation matrix of order 36 is directly related to the famous problem of 36 officers by Euler, and it follows from the nonexistence of two mutually orthogonal Latin Squares of size six. The more general question as to whether there exists a 2-unitary matrix of size $N^{2}=36$ (not necessarily a permutation) remains open [81].

Two-unitarity of a bipartite gate $U$ corresponds to a twouniform pure state of four parties maximally entangled with respect to all three partitions. Sometimes it is interesting to relax one requirement and analyze a pure state $\left|\psi_{A B C D}\right\rangle$ for which only two partial traces out of three are maximally mixed. This weaker condition corresponds to a unitary matrix $U$ of size $N^{2}$ such that additionally $U^{T_{A}}$ or $U^{R}$ is unitary. The class of unitary matrices such that the partial transposition $U^{T_{A}}$ remains unitary was studied in the context of quantum operations preserving some given matrix algebra, and a method to generate them numerically based on a kind of Sinkhorn algorithm was proposed [71,72]. Such a technique based on alternating projections on manifolds converges [86] if we wish to assure that two unitarity conditions are satisfied, so that two partial traces of the corresponding four-party state are fixed [87], but it will usually become less effective if three conditions (A4) need to be fulfilled simultaneously.

The observations made in this Appendix for the symmetric case, $M=N$, can be summarized as follows:

Proposition 1. For any unitary operator $U$ acting on a bipartite space $\mathcal{H}_{N}^{A} \otimes \mathcal{H}_{N}^{B}$, the following are equivalent.

(a) The unitary $U \in U\left(N^{2}\right)$ attains the global maximum of entangling power, that is, $e_{p}(U)=1$, as both linear entanglement entropies $E(U)$ and $E(U S)$ are maximal.

(b) The bipartite unitary matrix $U$ is 2-unitary. In other words, both the transformed matrices $U^{R}$ and $U^{T_{A}}$ remain unitary.

(c) If $U_{A B}=U$, the pure state

$$
\left|\psi_{A B C D}\right\rangle=\left(U_{A B} \otimes \mathbb{1}_{C D}\right)\left|\phi_{A C}^{+}\right\rangle \otimes\left|\phi_{B D}^{+}\right\rangle
$$


defined in Eq. (A1) is maximally entangled with respect to all possible bipartitions and thus forms an absolutely maximally entangled state of four quNits.

(d) The corresponding four-index tensor $u_{i \alpha, k \beta}$ whose elements describe the four-partite state

$$
\left|\psi_{A B C D}\right\rangle=\sum_{i, j=1}^{N} \sum_{\alpha, \beta=1}^{N} u_{i \alpha, j \beta}|i \alpha j \beta\rangle
$$

is perfect.

\section{APPENDIX B: THE STATIONARITY OF THE PARABOLA OF POWERS OF SWAP}

Lemma B.1. Define $f(u) \equiv e_{p}(u)-2 g_{t}(u)\left[1-g_{t}(u)\right]$, where $e_{p}(u)$ and $g_{t}(u)$ are, respectively, the entangling power and gate typicality of a bipartite unitary $u$. The function $f(u)$ is extremized whenever $u=U_{t}=e^{i t S}$ is a fractional power of the SWAP operator.

Proof. Operators close to arbitrary fractional powers of SWAP $U_{t}$, with $0<\epsilon \ll 1$, are

$$
U_{t, \epsilon}=\exp (i t S+i \epsilon H) \approx \exp (i t S)(\mathbb{1}+i \epsilon H),
$$

where $H$ is a Hermitian operator. We may require without loss of generality that $H$ is traceless, that is, $\operatorname{tr} H=0$, as the overall phase will make no difference to calculations. We may also assume that $H$ is orthogonal to $S$, that is, $\operatorname{tr}(H S)=\operatorname{tr}(S H)=$ 0 , as any overlap with $S$ will be equivalent to only shifting $t$ to a new value. The difference $\delta U_{t}=U_{t, \epsilon}-U_{t}$ is given by

$$
\delta U_{t}=i \epsilon(\cos t H+i \sin t S H) .
$$

We will show that $\delta E\left(U_{t}\right)=0$ and $\delta E\left(U_{t} S\right)=0$, thus under such perturbations $\delta e_{p}\left(U_{t}\right)=0$ and $\delta g_{t}\left(U_{t}\right)=0$ and finally $\delta f\left(u=U_{t}\right)=0$.

From Eq. (22) it follows that

$$
\delta E(U)=-\frac{4}{N^{2}} \operatorname{Re} \operatorname{tr}\left(\delta U^{R} U^{R \dagger} U^{R} U^{R \dagger}\right) .
$$

From the linearity of the reshuffling operation, $\delta U_{t}^{R}=$ $\left(U_{t, \epsilon}\right)^{R}-U_{t}^{R}=\left(\delta U_{t}\right)^{R}$. From this and Eq. (B2), we get

$$
\delta U_{t}^{R}=\left(\delta U_{t}\right)^{R}=i \epsilon\left[\cos t H^{R}+i \sin t(S H)^{R}\right] .
$$

To show that $\operatorname{tr}\left(\delta U_{t}^{R} U_{t}^{R \dagger} U_{t}^{R} U_{t}^{R \dagger}\right)=0$, we note that it involves $\operatorname{tr}\left(H^{R} \mathbb{1}_{R}\right), \operatorname{tr}\left(H^{R} S\right), \operatorname{tr}\left((S H)^{R} \mathbb{1}_{R}\right)$, and $\operatorname{tr}\left((S H)^{R} S\right)$. It is straightforward to verify that when $H$ is orthogonal to $S$ and is traceless, all of these vanish. In a similar way, it is easy to show also that $\operatorname{tr}\left(\delta U_{t}^{T_{A}} U_{t}^{T_{A} \dagger} U_{t}^{T} U_{t}^{T_{A} \dagger}\right)=0$. Thus $\delta f(u=$ $\left.U_{t}\right)=0$ when $u=U_{t}$ is a power of the SwAP $S$, except when $\delta u$ is along $S$. In the latter case, $f(u)=0$ strictly and there is no variation of $f$, establishing that $f(u)$ is indeed an extremum if $u=e^{i t S}$ is a fractional power of the SWAP.

\section{APPENDIX C: PROOF OF THE THEOREM CONCERNING AVERAGE ENTANGLING POWER $e_{p}\left[\mathcal{U}^{(n)}\right]$}

Theorem C.1. Let $U$ and $V$ be unitary operators on $\mathcal{H}_{N}^{A} \otimes$ $\mathcal{H}_{M}^{B}$, and let $u_{A}, u_{B}$ be sampled from the groups $U(N)$ and $U(M)$ of unitary matrices according to their Haar measures.
Then

$$
\begin{aligned}
&\left\langle e_{p}\right. {\left.\left[U\left(u_{A} \otimes u_{B}\right) V\right]\right\rangle_{u_{A}, u_{B}} } \\
& \quad=e_{p}(U)+e_{p}(V)-e_{p}(U) e_{p}(V) / \overline{e_{p}}
\end{aligned}
$$

where the average entangling power reads $\overline{e_{p}}=\left\langle e_{p}(W)\right\rangle_{W}$, and $W$ is sampled according to the Haar measure on the unitary group $U(N M)$.

Proof. Consider an extended Hilbert space $\mathcal{H}_{N}^{A} \otimes \mathcal{H}_{M}^{B} \otimes$ $\mathcal{H}_{A}^{C} \otimes \mathcal{H}_{M}^{D}$, where $\mathcal{H}_{N}^{C}$ and $\mathcal{H}_{M}^{D}$ are copies of $\mathcal{H}_{N}^{A}$ and $\mathcal{H}_{M}^{B}$. Using the identity $\operatorname{tr}\left(\rho_{A} \otimes \rho_{C} S_{A C}\right)=\operatorname{tr}\left(\rho_{A}^{2}\right)$, where $\rho_{C}$ is a copy of $\rho_{A}$, and $S_{A C}$ is the SWAP operator, the entangling power of $U$ acting on $\mathcal{H}_{N}^{A} \otimes \mathcal{H}_{M}^{B}$ was written in [9] as

$$
e_{p}(U)=\frac{2}{\tilde{e}_{p}^{\max }} \operatorname{tr}\left(U^{\otimes 2} \Omega_{p}^{++} U^{\dagger \otimes 2} \Pi_{A C}^{-}\right) .
$$

Here $\Pi_{A C}^{-}=2^{-1}\left(\mathbb{1}-S_{A C}\right)$ is the projector over the antisymmetric subspace of $\mathcal{H}_{N}^{A} \otimes \mathcal{H}_{N}^{C}$, and $\Omega_{p}^{++}=\omega_{A C}^{+} \otimes \omega_{B D}^{+}$ and $\omega_{A C}^{+}=\int d \mu\left(\psi_{A}\right)\left(\left|\psi_{A}\right\rangle\left\langle\psi_{A}|\otimes| \psi_{A}\right\rangle\left\langle\psi_{A}\right|\right)$, while $\omega_{B C}^{+}$is an identical operator. When $d \mu\left(\psi_{A}\right)$ is the Haar measure on states in $\mathcal{H}_{N}^{A}$, recognizing that $\omega_{A C}^{+}$has support only on the symmetric subspace, group theoretic arguments involving Schur's lemma were used in [9] to show that $\Omega_{p}^{++}=$ $4 C_{A} C_{B} \Pi_{A C}^{+} \Pi_{B D}^{+}$. Here $C_{A}^{-1}=N(N+1), C_{B}^{-1}=M(M+1)$, $\Pi_{A C}^{+}=2^{-1}\left(\mathbb{1}+S_{A C}\right)$ is the projector over the symmetric subspace of $\mathcal{H}_{N}^{A} \otimes \mathcal{H}_{N}^{C}$, while $\Pi_{B D}^{+}$is a similar projector on $\mathcal{H}_{M}^{B} \otimes \mathcal{H}_{M}^{D}$.

This forms a convenient starting point for us, as the local unitary averaged entangling power is

$$
\left\langle e_{p}\left[U\left(u_{A} \otimes u_{B}\right) V\right]\right\rangle_{u_{A}, u_{B}}=\frac{2}{\tilde{e}_{p}^{\max }} \operatorname{tr}\left(U^{\otimes 2}\langle Q\rangle U^{\dagger \otimes 2} \Pi_{A C}^{-}\right),
$$

where $Q=V \otimes V \Omega_{p}^{++} V^{\dagger} \otimes V^{\dagger}$, and

$$
\begin{aligned}
\langle Q\rangle= & \int d \mu\left(u_{A}\right) d \mu\left(u_{B}\right)\left(u_{A} \otimes u_{B}\right)^{\otimes 2} \\
& \times V^{\otimes 2} \Omega_{p}^{++} V^{\dagger \otimes 2}\left(u_{A}^{\dagger} \otimes u_{B}^{\dagger}\right)^{\otimes 2} .
\end{aligned}
$$

Since the local unitaries are sampled independently, the average over $u_{A}, u_{B}$ can be done separately. Note that $V \otimes V$ acts on $A B$ and its copy $C D$, while $\Omega_{p}^{++}$acts on $A C$ and $B D$ independently. Note also that $\langle Q\rangle$ is self-adjoint and hence diagonalizable. For any $x_{A} \in U(N),\left[\left(x_{A}\right)^{\otimes 2},\langle Q\rangle\right]=0$ due to the unitary invariance of the Haar measure. With similar reasoning $\left[\left(x_{B}\right)^{\otimes 2},\langle Q\rangle\right]=0, \forall x_{B} \in U(M)$. Since $\left(x_{A}\right)^{\otimes 2},\left(x_{B}\right)^{\otimes 2}$ act irreducibly on the totally symmetric and antisymmetric subspaces, it follows from the above commutation relations and Schur's lemma [88] that $\langle Q\rangle$ can be written as a linear combination of projectors on the symmetric and antisymmetric subspaces,

$$
\begin{aligned}
\langle Q\rangle= & \alpha_{1} \Pi_{A C}^{+} \Pi_{B D}^{+}+\alpha_{2} \Pi_{A C}^{-} \Pi_{B D}^{-} \\
& +\alpha_{3} \Pi_{A C}^{+} \Pi_{B D}^{-}+\alpha_{4} \Pi_{A C}^{-} \Pi_{B D}^{+},
\end{aligned}
$$

where $\alpha_{l}=\left[\operatorname{tr}\left(\Pi_{A C}^{ \pm} \Pi_{B D}^{ \pm}\right)\right]^{-1} \operatorname{tr}\left(Q \Pi_{A C}^{ \pm} \Pi_{B D}^{ \pm}\right) ; l=\{1, \ldots, 4\}$. That the operator $Q$ can be used for finding $\alpha_{l}$ instead of $\langle Q\rangle$ follows from the fact that $\left(u_{A}^{\dagger} \otimes u_{C}^{\dagger}\right) \Pi_{A C}^{+}\left(u_{A} \otimes u_{C}\right)=\Pi_{A C}^{+}$. 
Next, we evaluate expressions for $\operatorname{tr}(Q)$ and $\operatorname{tr}\left(Q S_{A C}\right)$ as follows (summation over repeated indices is assumed):

$$
\begin{aligned}
\operatorname{tr}(Q)= & \operatorname{tr}\left(\Omega_{p}^{++}\right)=1 \\
& \times \operatorname{tr}\left(Q S_{A C} \otimes S_{B D}\right) \\
= & \frac{1}{N(N+1)} \frac{1}{M(M+1)} \operatorname{tr}\left[V \otimes V \left(1+S_{A C}+S_{B D}\right.\right. \\
& \left.\left.+S_{A C} \otimes S_{B D}\right) V^{\dagger} \otimes V^{\dagger} S_{A C} \otimes S_{B D}\right] .
\end{aligned}
$$

Now,

$$
\begin{aligned}
\operatorname{tr} & \left(V \otimes V S_{A C} V^{\dagger} \otimes V^{\dagger} S_{A C} \otimes S_{B D}\right) \\
& =\left\langle i_{1} \alpha_{1} j_{1} \beta_{1}|V \otimes V| i_{2} \alpha_{2} j_{2} \beta_{2}\right\rangle\left\langle j_{2} \alpha_{2} i_{2} \beta_{2}\left|V^{\dagger} \otimes V^{\dagger}\right| j_{2} \beta_{2} i_{2} \alpha_{2}\right\rangle \\
& =N^{2} M .
\end{aligned}
$$

Similarly,

$$
\begin{aligned}
\operatorname{tr}\left(V \otimes V S_{B D} V^{\dagger} \otimes V^{\dagger} S_{A C} \otimes S_{B D}\right) & =N M^{2}, \\
\operatorname{tr}\left(V \otimes V S_{A C} \otimes S_{B D} V^{\dagger} \otimes V^{\dagger} S_{A C} \otimes S_{B D}\right) & =N^{2} M^{2} .
\end{aligned}
$$

Combining these trace relations in Eq. (C6) gives

$$
\operatorname{tr}\left(Q S_{A C} \otimes S_{B D}\right)=1 .
$$

To compute $\operatorname{tr}\left(Q S_{A C}\right)$ and $\operatorname{tr}\left(Q S_{B D}\right)$, note that

$$
\begin{aligned}
\operatorname{tr}\left(\rho_{A C}^{2}\right) & =\frac{1}{N^{2} M^{2}} \operatorname{tr}\left[\left[V^{R}\left(V^{R}\right)^{\dagger}\right]^{2}\right] \\
& =\frac{1}{N^{2} M^{2}} \operatorname{tr}\left(V \otimes V S_{A C} V^{\dagger} \otimes V^{\dagger} S_{A C}\right),
\end{aligned}
$$

where the equality in the second line can be seen via a similar calculation as in Eq. (C7) (see also [89]). Similarly,

$$
\begin{aligned}
\operatorname{tr}\left(\rho_{A D}^{2}\right) & =\frac{1}{N^{2} M^{2}} \operatorname{tr}\left[\left[V^{T_{A}}\left(V^{T_{A}}\right)^{\dagger}\right]^{2}\right] \\
& =\frac{1}{N^{2} M^{2}} \operatorname{tr}\left(V \otimes V S_{B D} V^{\dagger} \otimes V^{\dagger} S_{A C}\right) .
\end{aligned}
$$

Using Eqs. (45), (C10), and (C11),

$\operatorname{tr}\left(Q S_{A C}\right)$

$$
\begin{aligned}
= & \frac{1}{N(N+1)} \frac{1}{M(M+1)} \operatorname{tr}\left[V \otimes V \left(1+S_{A C}+S_{B D}\right.\right. \\
& \left.\left.+S_{A C} \otimes S_{B D}\right) V^{\dagger} \otimes V^{\dagger} S_{A C}\right] \\
= & \frac{1}{N(N+1)} \frac{1}{M(M+1)} \operatorname{tr}\left(N M^{2}+N^{2} M\right. \\
& \left.+\operatorname{tr}\left[V^{R}\left(V^{R}\right)^{\dagger}\right]^{2}+\operatorname{tr}\left[V^{T_{A}}\left(V^{T_{A}}\right)^{\dagger}\right]^{2}\right) \\
= & 1-\tilde{e}_{p}^{\max } e_{p}(V) .
\end{aligned}
$$

Similarly,

$$
\operatorname{tr}\left(Q S_{A C}\right)=1-\tilde{e}_{p}^{\max } e_{p}(V) .
$$

Using Eq. (C3) and the traces evaluated above, we get

$$
\begin{aligned}
\operatorname{tr}(Q) & =\operatorname{tr}\left[Q\left(S_{A C} \otimes S_{B D}\right)\right]=1, \\
\operatorname{tr}\left(Q S_{A C}\right) & =\operatorname{tr}\left(Q S_{B D}\right)=1-\tilde{e}_{p}^{\max } e_{p}(V) .
\end{aligned}
$$
by

Thus the local unitary averaged entangling power is given

$$
\left\langle e_{p}\left[U\left(u_{A} \otimes u_{B}\right) V\right]\right\rangle_{u_{A}, u_{B}}=e_{p}(U)+\left[1-\frac{e_{p}(U)}{\overline{e_{p}}}\right] e_{p}(V),
$$

where $\overline{e_{p}}$ is the CUE averaged entangling power in Eq. (47).

Corollary C.1.1. The local unitary averaged gate typicality is [44]

$$
\left\langle g_{t}\left(U^{(n)}\right)\right\rangle_{\mathrm{loc}}=\bar{g}_{t}\left\{1-\left[1-g_{t}(U) / \bar{g}_{t}\right]^{n}\right\},
$$

where the average value $\bar{g}_{t}$ is given in Eq. (47).

Proof. When $M=N$, gate typicality in Eq. (18) is given by

$$
g_{t}(U)=\operatorname{tr}\left(U^{\otimes 2} \Omega_{p}^{+-} U^{\dagger \otimes 2} \Pi_{A C}^{-}\right),
$$

where $\Omega_{p}^{+-}=C_{A} \tilde{C}_{B} \Pi_{A C}^{+} \Pi_{B D}^{-}, \tilde{C}_{B}^{-1}=N(N-1)$. Starting with the above relation for $M \neq N$ and proceeding in the same way as in the proof of entangling power proves the corollary.
[1] B. Swingle, G. Bentsen, M. Schleier-Smith, and P. Hayden, Phys. Rev. A 94, 040302(R) (2016).

[2] A. Nahum, J. Ruhman, S. Vijay, and J. Haah, Phys. Rev. X 7, 031016 (2017).

[3] R.-Q. He and Z.-Y. Lu, Phys. Rev. B 95, 054201 (2017).

[4] T. Zhou and D. J. Luitz, Phys. Rev. B 95, 094206 (2017).

[5] A. Seshadri, V. Madhok, and A. Lakshminarayan, Phys. Rev. E 98, 052205 (2018).

[6] P. Hosur, X.-L. Qi, D. A. Roberts, and B. Yoshida, J. High Energy Phys. 02 (2016) 004.

[7] A. Chan, A. De Luca, and J. T. Chalker, Phys. Rev. X 8, 041019 (2018).

[8] C. W. von Keyserlingk, T. Rakovszky, F. Pollmann, and S. L. Sondhi, Phys. Rev. X 8, 021013 (2018).

[9] P. Zanardi, C. Zalka, and L. Faoro, Phys. Rev. A 62, 030301(R) (2000).

[10] P. Zanardi, Phys. Rev. A 63, 040304(R) (2001).
[11] X. Wang and P. Zanardi, Phys. Rev. A 66, 044303 (2002).

[12] K. Życzkowski and I. Bengtsson, Open Syst. Inf. Dyn. 11, 3 (2004).

[13] J. Dubail, J. Phys. A 50, 234001 (2017).

[14] R. Pal and A. Lakshminarayan, Phys. Rev. B 98, 174304 (2018).

[15] E. Lubkin and T. Lubkin, Int. J. Theor. Phys. 32, 933 (1993).

[16] P. A. Miller and S. Sarkar, Phys. Rev. E 60, 1542 (1999).

[17] J. N. Bandyopadhyay and A. Lakshminarayan, Phys. Rev. Lett. 89, 060402 (2002).

[18] H. Fujisaki, T. Miyadera, and A. Tanaka, Phys. Rev. E 67, 066201 (2003).

[19] J. N. Bandyopadhyay and A. Lakshminarayan, Phys. Rev. E 69, 016201 (2004)

[20] R. Demkowicz-Dobrzański and M. Kuś, Phys. Rev. E 70, 066216 (2004).

[21] N. Linden, S. Popescu, A. J. Short, and A. Winter, Phys. Rev. E 79, 061103 (2009). 
[22] S. Chaudhury, A. Smith, B. E. Anderson, S. Ghose, and P. S. Jessen, Nature (London) 461, 768 (2009).

[23] C. Neill et al., Nat. Phys. 12, 1037 (2016).

[24] A. Lakshminarayan, S. C. L. Srivastava, R. Ketzmerick, A. Bäcker, and S. Tomsovic, Phys. Rev. E 94, 010205(R) (2016).

[25] N. Schuch, M. M. Wolf, K. G. H. Vollbrecht, and J. I. Cirac, New J. Phys. 10, 033032 (2008).

[26] R. F. Abreu and R. O. Vallejos, Phys. Rev. A 75, 062335 (2007).

[27] P. Calabrese, Physica A 504, 31 (2018), lecture notes of the 14th International Summer School on Fundamental Problems in Statistical Physics.

[28] B. Bertini, P. Kos, and T. Prosen, Phys. Rev. X 9, 021033 (2019).

[29] S. Bravyi, Phys. Rev. A 76, 052319 (2007).

[30] C. Petitjean and P. Jacquod, Phys. Rev. Lett. 97, 194103 (2006).

[31] C. M. Trail, V. Madhok, and I. H. Deutsch, Phys. Rev. E 78, 046211 (2008).

[32] J. J. Pulikkottil, A. Lakshminarayan, S. C. L. Srivastava, A. Bäcker, and S. Tomsovic, Phys. Rev. E 101, 032212 (2020).

[33] S. Moudgalya, T. Devakul, C. W. von Keyserlingk, and S. L. Sondhi, Phys. Rev. B 99, 094312 (2019).

[34] M. A. Nielsen and I. L. Chuang, Quantum Computation and Quantum Information (Cambridge University Press, Cambridge, 2000).

[35] J. Emerson, Y. S. Weinstein, M. Saraceno, S. Lloyd, and D. G. Cory, Science 302, 2098 (2003).

[36] A. W. Harrow and R. A. Low, Commun. Math. Phys. 291, 257 (2009).

[37] P. Kondratiuk and K. Życzkowski, Acta Phys. Pol. A 124, 1098 (2013).

[38] C. Dankert, R. Cleve, J. Emerson, and E. Livine, Phys. Rev. A 80, 012304 (2009).

[39] F. G. S. L. Brandao, A. W. Harrow, and M. Horodecki, Commun. Math. Phys. 346, 397 (2016).

[40] J. Emerson, R. Alicki, and K. Życzkowski, J. Opt. B 7, S347 (2005).

[41] C. Sünderhauf, D. Pérez-García, D. A. Huse, N. Schuch, and J. I. Cirac, Phys. Rev. B 98, 134204 (2018).

[42] B. Collins, I. Nechita, and K. Życzkowski, J. Phys. A 43, 275303 (2010).

[43] S. K. Mishra and A. Lakshminarayan, Europhys. Lett. 105, 10002 (2014)

[44] B. Jonnadula, P. Mandayam, K. Życzkowski, and A. Lakshminarayan, Phys. Rev. A 95, 040302(R) (2017).

[45] A. Mandarino, T. Linowski, and K. Życzkowski, Phys. Rev. A 98, 012335 (2018).

[46] A. Higuchi and A. Sudbery, Phys. Lett. A 273, 213 (2000).

[47] V. L. Girko, Theor. Probab. Appl. 29, 694 (1985).

[48] V. A. Marčenko and L. A. Pastur, Math. USSR Sb. 1, 457 (1967).

[49] M. Musz, M. Kuś, and K. Życzkowski, Phys. Rev. A 87, 022111 (2013).

[50] K. Życzkowski and H.-J. Sommers, J. Phys. A: Math. Gen. 34, 7111 (2001).

[51] M. Akila, D. Waltner, B. Gutkin, and T. Guhr, J. Phys. A 49, 375101 (2016).

[52] B. Bertini, P. Kos, and T. Prosen, Phys. Rev. Lett. 123, 210601 (2019).
[53] M. A. Nielsen, C. M. Dawson, J. L. Dodd, A. Gilchrist, D. Mortimer, T. J. Osborne, M. J. Bremner, A. W. Harrow, and A. Hines, Phys. Rev. A 67, 052301 (2003).

[54] B. Bertini, P. Kos, and T. Prosen, SciPost Phys. 8, 067 (2020).

[55] B. Gutkin, P. Braun, M. Akila, D. Waltner, and T. Guhr, arXiv:2001.01298.

[56] L. Piroli, B. Bertini, J. I. Cirac, and T. Prosen, Phys. Rev. B 101, 094304 (2020).

[57] S. A. Rather, S. Aravinda, and A. Lakshminarayan, Phys. Rev. Lett. 125, 070501 (2020).

[58] D. Goyeneche, D. Alsina, J. I. Latorre, A. Riera, and K. Życzkowski, Phys. Rev. A 92, 032316 (2015).

[59] W. Helwig, W. Cui, J. I. Latorre, A. Riera, and H. K. Lo, Phys. Rev. A 86, 052335 (2012).

[60] T. Linowski, G. Rajchel-Mieldzioć, and K. Życzkowski, J. Phys. A 53, 125303 (2020).

[61] The statement extends to any $U \in U(4)$, since any bipartite unitary $U \in U(4)$ can be expressed as the product of a $U \in S U$ (4) and a global phase shift $e^{i \alpha}$.

[62] N. Khaneja, R. Brockett, and S. J. Glaser, Phys. Rev. A 63, 032308 (2001).

[63] B. Kraus and J. I. Cirac, Phys. Rev. A 63, 062309 (2001).

[64] J. Zhang, J. Vala, S. Sastry, and K. B. Whaley, Phys. Rev. A 67, 042313 (2003).

[65] A. T. Rezakhani, Phys. Rev. A 70, 052313 (2004).

[66] S. Balakrishnan and R. Sankaranarayanan, Phys. Rev. A 79, 052339 (2009).

[67] S. Balakrishnan and R. Sankaranarayanan, Phys. Rev. A 83 062320 (2011).

[68] D. Collins, N. Linden, and S. Popescu, Phys. Rev. A 64, 032302 (2001).

[69] L. Clarisse, S. Ghosh, S. Severini, and A. Sudbery, Phys. Rev. A 72, 012314 (2005).

[70] M. Poźniak, K. Życzkowski, and M. Kuś, J. Phys. A: Math. Gen. 31, 1059 (1998).

[71] J. Deschamps, I. Nechita, and C. Pellegrini, J. Phys. A 49, 335301 (2016).

[72] T. Benoist and I. Nechita, Lin. Alg. Appl. 521, 70 (2017).

[73] F. Vatan and C. Williams, Phys. Rev. A 69, 032315 (2004).

[74] S. C. L. Srivastava, S. Tomsovic, A. Lakshminarayan, R. Ketzmerick, and A. Bäcker, Phys. Rev. Lett. 116, 054101 (2016).

[75] S. Tomsovic, A. Lakshminarayan, S. C. L. Srivastava, and A. Bäcker, Phys. Rev. E 98, 032209 (2018).

[76] A. Lakshminarayan, Z. Puchała, and K. Życzkowski, Phys. Rev. A 90, 032303 (2014).

[77] Z. D. Bai, Ann. Probab. 25, 494 (1997).

[78] J. A. Mingo, M. Popa, and K. Szpojankowski, arXiv:2006.05408.

[79] S. M. Cohen and L. Yu, Phys. Rev. A 87, 022329 (2013).

[80] F. Haake, M. Kuś, H.-J.Sommers, H. Schomerus, and K. Życzkowski, J. Phys. A: Math. Gen. 29, 3461 (1996).

[81] P. Horodecki, Ł. Rudnicki, and K. Życzkowski, arXiv:2002.03233.

[82] F. Haake, S. Gnutzmann, and M. Kuś, Quantum Quantum Signatures of Chaos, 4th ed. (Springer, Berlin, 2018).

[83] I. Bengtsson and K. Życzkowski, Geometry of Quantum States: An Introduction to Quantum Entanglement (Cambridge University Press, Cambridge, 2007). 
[84] F. Pastawski, B. Yoshida, D. Harlow, and J. Preskill, J. High Energy Phys. 06 (2015) 149.

[85] A. J. Scott, Phys. Rev. A 69, 052330 (2004).

[86] A. Lewis and J. Malick, Math. Oper. Res. 33, 216 (2008).
[87] X. Duan, C.-K. Li, and D. C. Pelejo, arXiv:1604.08289.

[88] J. F. Cornwell, Group Theory in Physics: An Introduction (Academic Press, 1997).

[89] X. Wang, B. C. Sanders, and D. W. Berry, Phys. Rev. A 67, 042323 (2003). 